\title{
Arabidopsis phosphatidylinositol monophosphate 5-kinase 2 is involved in root gravitropism through regulation of polar auxin transport by affecting the cycling of PIN proteins
}

\author{
Yu Mei ${ }^{1, *}$, Wen-Jing Jia ${ }^{1, *}$, Yu-Jia Chu ${ }^{1}$, Hong-Wei Xue \\ ${ }^{1}$ National Key Laboratory of Plant Molecular Genetics, Institute of Plant Physiology \& Ecology, Shanghai Institutes for Biological \\ Sciences, Chinese Academy of Sciences, 300 Fenglin Road, Shanghai 200032, China
}

Phosphatidylinositol monophosphate 5-kinase (PIP5K) catalyzes the synthesis of PI-4,5-bisphosphate $(\operatorname{PtdIns}(4,5)$ $P_{2}$ ) by phosphorylation of PI-4-phosphate at the 5 position of the inositol ring, and is involved in regulating multiple developmental processes and stress responses. We here report on the functional characterization of Arabidopsis PIP5K2, which is expressed during lateral root initiation and elongation, and whose expression is enhanced by exogenous auxin. The knockout mutant pip $5 k 2$ shows reduced lateral root formation, which could be recovered with exogenous auxin, and interestingly, delayed root gravity response that could not be recovered with exogenous auxin. Crossing with the DR5-GUS marker line and measurement of free IAA content confirmed the reduced auxin accumulation in pip5k2. In addition, analysis using the membrane-selective dye FM4-64 revealed the decelerated vesicle trafficking caused by PtdIns(4,5) $\mathrm{P}_{2}$ reduction, which hence results in suppressed cycling of PIN proteins (PIN2 and $3)$, and delayed redistribution of PIN2 and auxin under gravistimulation in pip5k2 roots. On the contrary, PtdIns $(4,5)$ $P_{2}$ significantly enhanced the vesicle trafficking and cycling of PIN proteins. These results demonstrate that PIP5K2 is involved in regulating lateral root formation and root gravity response, and reveal a critical role of PIP5K2/PtdIns $(4,5) \mathrm{P}_{2}$ in root development through regulation of PIN proteins, providing direct evidence of crosstalk between the phosphatidylinositol signaling pathway and auxin response, and new insights into the control of polar auxin transport.

Keywords: Arabidopsis; phosphatidylinositol monophosphate 5-kinase 2 (PIP5K2); root development; polar auxin transport; vesicle trafficking

Cell Research (2012) 22:581-597. doi:10.1038/cr.2011.150; published online 6 September 2011

\section{Introduction}

The phosphatidylinositol (PI) signaling pathway and its relevant molecules play critical roles in regulating plant growth and stress response (for reviews, [1-3]). PI monophosphate 5-kinase (PIP5K) is a key enzyme in the PI signaling pathway that catalyzes the synthesis of

\footnotetext{
*These two authors contributed equally to the work.

Correspondence: Hong-Wei Xue

Tel: +86-21-54924059; Fax: +86-21-54924060

E-mail: hwxue@sibs.ac.cn

Received 4 July 2011; revised 22 July 2011; accepted 25 July 2011; published online 6 September 2011
}

PI-4,5-bisphosphate $\left[\operatorname{PtdIns}(4,5) \mathrm{P}_{2}\right]$, which is the precursor of two important secondary messengers: inositol 1,4,5-trisphosphate $\left[\operatorname{Ins}(1,4,5) \mathrm{P}_{3}\right]$ and diacylglycerol $[4$, $5]$.

As a multifunctional molecule, the role of $\operatorname{Ptdnns}(4,5)$ $\mathrm{P}_{2}$ is far beyond simple conversion to its end products, and research in animal cells has shown that PtdIns $(4,5)$ $\mathrm{P}_{2}$ is involved in various processes via interactions with different protein partners, including actin-interacting enzymes (for review, $[6,7]$ ). In addition, PIP5K and its product PtdIns $(4,5) \mathrm{P}_{2}$ play critical roles in both anterograde and retrograde vesicle trafficking. PtdIns $(4,5) \mathrm{P}_{2}$ can bind many endocytic proteins and affects endocytosis by modulating the actin cytoskeleton assembly, or 
plays multiple roles in exocytosis by binding to several exocyst components. PtdIns $(4,5) \mathrm{P}_{2}$ can also interact with ion channels (for review, $[8,9]$ ) and regulators of vesicle trafficking in plant (for reviews, [10-12]).

In Arabidopsis thaliana, 15 putative proteins that have significant sequence similarity to animal or yeast PIP5Ks [13] have been identified. Besides 4 homologs of yeast PIP5K, 11 PIPK isoforms can be divided into two subfamilies (A and $\mathrm{B}$ ) according to their domain organization [14]. The catalytic activities of using PtdIns4P as the preferred substrate to generate $\operatorname{PtdIns}(4,5) \mathrm{P}_{2}$ have all been characterized [15-18]. Studies have revealed the physiological functions of some PIP5Ks, including PIP5K1 in abscisic acid signaling [19]; PIP5K3 in root hair formation and growth $[16,20]$; PIP5K4 in stomata opening [21]; and PIP5K4-6 in pollen tube growth [17, 22]. In addition, PIP5K9 regulates root growth and is involved in sugar signaling [23].

Root architecture determines the ability of a plant to acquire water and nutrients from the soil. Lateral roots in Arabidopsis are derived from a subset of pericycle founder cells, which are adjacent to the two xylem poles. Upon initiation, the pericycle cells divide and develop lateral root apical meristems, which in turn produce mature lateral roots. Among the signals that determine lateral root formation, the plant hormone auxin is widely considered a key regulator [24, 25]. Arabidopsis mutants with high levels of endogenous auxin as well as seedlings treated with exogenous auxin display increased lateral roots [2628], whereas mutants defective in auxin accumulation, transport and signaling produce fewer lateral roots [2931].

Root gravitropism is also regulated by auxin, especially polar auxin transport, which is mediated by membranelocalized auxin-influx and -efflux carriers, including AUX1/LAX proteins, the PIN-FORMED (PIN) efflux carriers and the MULTIDRUG RESISTANCE/P-GLYCOPROTEIN (PGP) class of ATP-binding cassette auxin transporters [32-35]. Genetic analyses have revealed the critical roles of the influx carrier AUX1 and efflux carrier PIN proteins in gravity response. Mutation in AUXI abolishes root gravitropic curvature [36]; similarly, pin2 seedlings are agravitropic, whereas PIN2 overexpression results in an enhanced tropistic response [37, 38]. The lateral relocation of PIN3 in response to gravity redirects the auxin flux and mediates tropism in Arabidopsis [39]. Correlation of PI signaling and PIPK activity with gravitropism was first discovered in maize pulvini, of which the rapid changes of the specific activity of PIP5K and levels of the second messenger Ins $(1,4,5) \mathrm{P}_{3}$ were observed in the early phase of gravistimulation [40]. Later, the universal role of $\operatorname{Ins}(1,4,5) \mathrm{P}_{3}$ in the gravity response was further revealed in Arabidopsis [41, 42].

Recent studies have shown that PIN proteins (PIN1, 2 and 3) undergo a constitutive cycling between the plasma membrane and endosomal compartments, which is of great importance to their polar subcellular localization and function $[39,43]$. This process is dependent on clathrin-mediated endocytosis, intracellular vesicle trafficking [44, 45] and actin cytoskeleton, and is controlled by auxin signaling and membrane sterols [12, 46-48].

Although many pieces of evidence have indicated the crosstalk between the PI signaling pathway and auxin signaling pathway via regulation of auxin homeostasis or auxin transport [12, 42, 49-51], the potential link of PIP5K with auxin-related processes has not yet been directly investigated. Here we report on the functional characterization of Arabidopsis PIP5K2, which is expressed in various tissues and whose expression is enhanced by exogenous auxin. Our studies indicate that PIP5K2 participates in both lateral root formation and root gravity response of Arabidopsis by regulating auxin accumulation and polar auxin transport.

\section{Results}

Structural analysis of Arabidopsis PIP5K2, which is expressed in various tissues and whose expression is enhanced by exogenous IAA

To study the physiological function of Arabidopsis PIP5K2, the full-length cDNA of PIP5K2 was amplified by PCR, which is 2265 -bp long and encodes a 754 -amino-acid peptide $(\sim 86.3 \mathrm{kDa})$ with high similarity to other type B PIP5Ks in Arabidopsis. Structural organization analysis indicated that PIP5K2 contains seven MORN (membrane occupation and recognition nexus) motifs at the $\mathrm{N}$ terminus (residues 80 - 239) and a catalytic domain at the $\mathrm{C}$ terminus (residues 382 - 751; Figure 1A).

Study of the expression pattern of PIP 5K2 by quantitative real-time RT-PCR (qRT-PCR) analysis showed that PIP5K2 was transcribed in various Arabidopsis tissues, including roots, shoots, leaves, flowers and seedlings (Figure 1B). Further, the detailed expression pattern of PIP5K2 was investigated by promoter-reporter gene fusion studies. The $1.9-\mathrm{kb}$ promoter region of PIP $5 K 2$ was amplified and fused to the GUS coding region, and then introduced into Arabidopsis. Analysis of GUS activities of independent positive transgenic lines revealed that PIP5K2 was highly expressed in young seedlings, including the cotyledons, hypocotyls, roots (Figure 1C, a), flowers, leaves and siliques, but not in the developing seeds (Figure $1 \mathrm{C}, \mathrm{b}-\mathrm{e}$ ). Strong expression of PIP5K2 was detected in the central cylinder, lateral root primordia and emerged lateral roots at different developmen- 


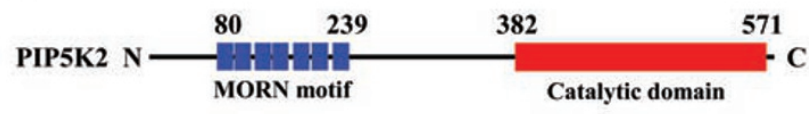

C
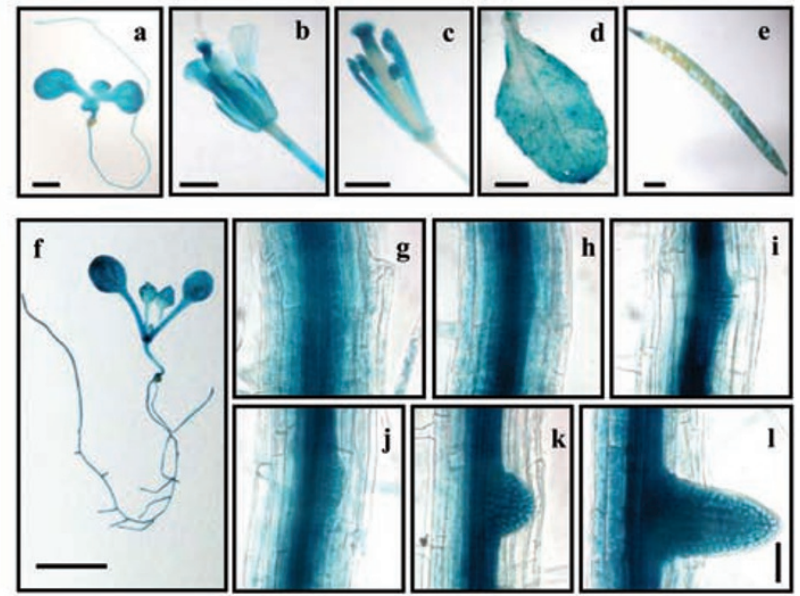

B

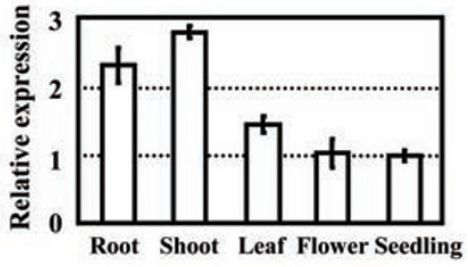

D
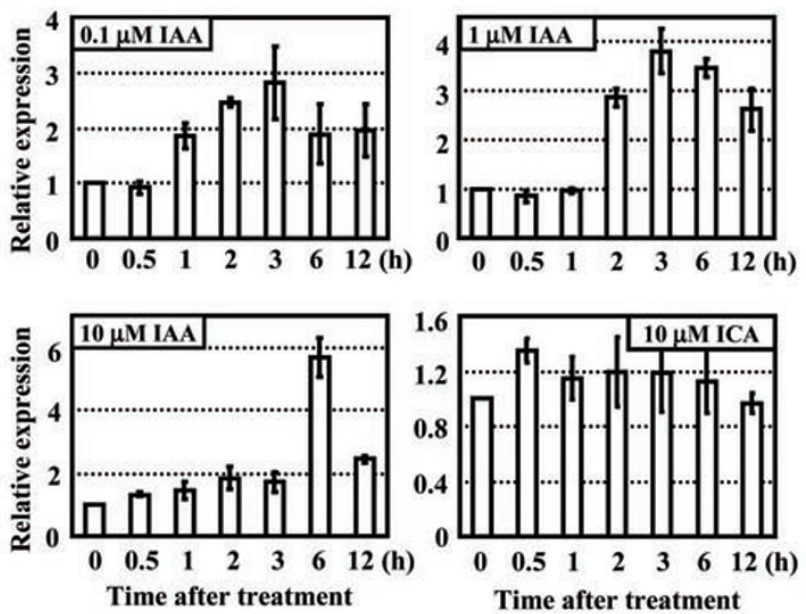

Figure 1 Structural organization and expression pattern of Arabidopsis PIP5K2. (A) Structural organization of Arabidopsis PIP5K2. Seven MORN motifs and the catalytic domain are localized at the $\mathrm{N}$ and $\mathrm{C}$ terminus of PIP5K2, respectively. (B) qRT-PCR analysis of PIP5K2 expression in various tissues. The ACTIN7 gene was used as an internal positive control and the transcript level of PIP5K2 in seedlings was set as 1.0. Error bars represent SD $(n=3)$. (C) Promoter-GUS fusion analysis of the expression of PIP5K2. Studies reveal the expression of PIP5K2 in young seedlings (a), floral tissues (b, c), leaves (d) and siliques (e). Strong expression of PIP5K2 is detected in the central cylinder, lateral root primordia and emerged lateral roots at developmental stage A (g, h), B (i-k), C (I) and D (f). The developmental stages were classified according to Zhang et al. [53] (same for Figure 2C). Scale bar $=1 \mathrm{~mm}(\mathrm{a}-\mathrm{e}), 0.5 \mathrm{~cm}$ (f) or $50 \mu \mathrm{m}$ (g-l). (D) qRT-PCR analysis of PIP5K2 expression after treatment with exogenous IAA. Ten-day-old Arabidopsis seedlings were treated with IAA (0.1, 1 or $10 \mu \mathrm{M})$ or indole-3carboxylic acid (ICA, $10 \mu \mathrm{M}$ ) for $0,0.5,1,2,3,6$ or $12 \mathrm{~h}$. The ACTIN7 gene was used as an internal positive control and the transcript level of PIP5K2 in untreated seedlings was set as 1.0. Error bars represent SD $(n=3)$.

tal stages (Figure 1C, f-1). Compared to the promoterreporter study of PIP5K2 performed by Stenzel et al. [16], our results provide more detailed expression patterns of PIP5K2, especially in floral tissues and during lateral root development.

qRT-PCR analysis further revealed that PIP5K2 expression is enhanced by exogenous IAA. Elevated transcript levels of PIP5K2 were detected under IAA treatment $(0.1,1$ or $10 \mu \mathrm{M})$, while the expression remained unaffected upon treatment with indole-3-carboxylic acid (ICA), a structural analog of IAA with no biological activity (Figure 1D). According to these results, we speculated that PIP5K2 may play a role in auxin-related processes.
PIP5K2 knockout results in reduced lateral root formation

To study the physiological functions of PIP5K2, a putative knockout mutant pip5k2 (SALK_012487) was identified by searching the Salk Institute T-DNA insertion library database (http://signal.salk.edu/cgi-bin/ tdnaexpress, [52]). The T-DNA was inserted at the first intron (Figure 2A, upper panel), which was confirmed by PCR analysis. The F2 progeny of heterozygous mutant plants segregated at a 3:1 ratio, indicating a single T-DNA insertion in the genome. Furthermore, qRT-PCR analysis revealed the deficiency of PIP5K2 expression in homozygous plants (Figure 2A, lower panel).

Phenotypic observation showed that the pip $5 \mathrm{k} 2 \mathrm{mu}-$ tant plants have normal appearance, including growth 
rate, flowering and fertility when grown under standard growth conditions; however, the lateral root number of pip $5 k 2$ was much reduced compared to the wild type (WT) when the seedlings were grown on vertical MS medium for 10-12 days (Figure 2B). To further investigate the reason for the reduced number of lateral roots, the numbers of lateral root primordia and lateral roots at different developmental stages were counted and statistically analyzed. The developmental stages of lateral roots were classified according to Zhang et al. [53] (stage A, up to three cell layers; stage B, unemerged lateral root of more than three cell layers; stage $\mathrm{C}$, emerged lateral root less than $0.5 \mathrm{~mm}$ in length; stage $\mathrm{D}$, lateral root longer than $0.5 \mathrm{~mm}$ ). The numbers of lateral root primordia and lateral roots of all four stages of pip $5 \mathrm{k} 2$ seedlings were significantly reduced compared to WT. The reduction was more severe in developmental stages A and D (Figure $2 \mathrm{C}$ ), indicating that the reduced number of lateral roots in pip $5 \mathrm{k} 2$ is more likely caused by reduced lateral root initiation rather than defect in lateral root emergence with normal initiation.

To confirm whether the pip $5 k 2$ phenotype is indeed caused by PIP5K2 deficiency, the coding region of $P I P 5 K 2$ driven by the CaMV35S promoter was transferred into the pip $5 k 2$ plants through genetic transformation. Homozygous lines containing single-copy TDNA insertions were obtained and used for subsequent analysis. The recovered expression levels of PIP5K2 in
A

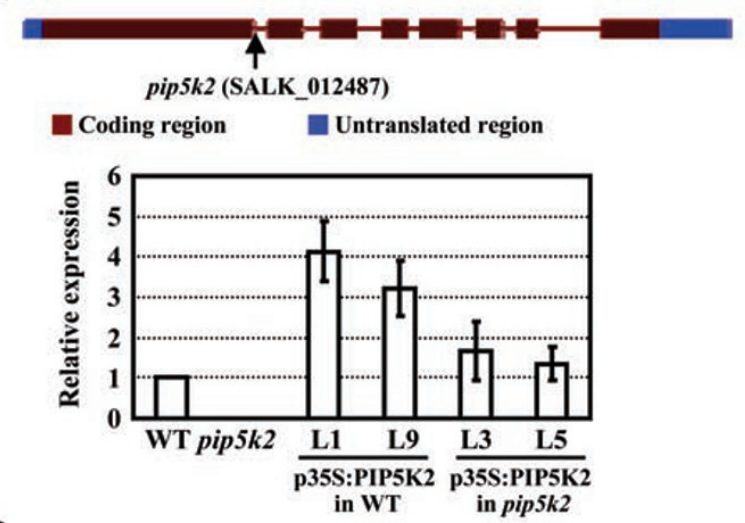

B

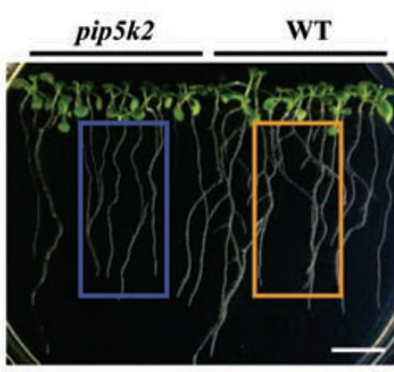

C
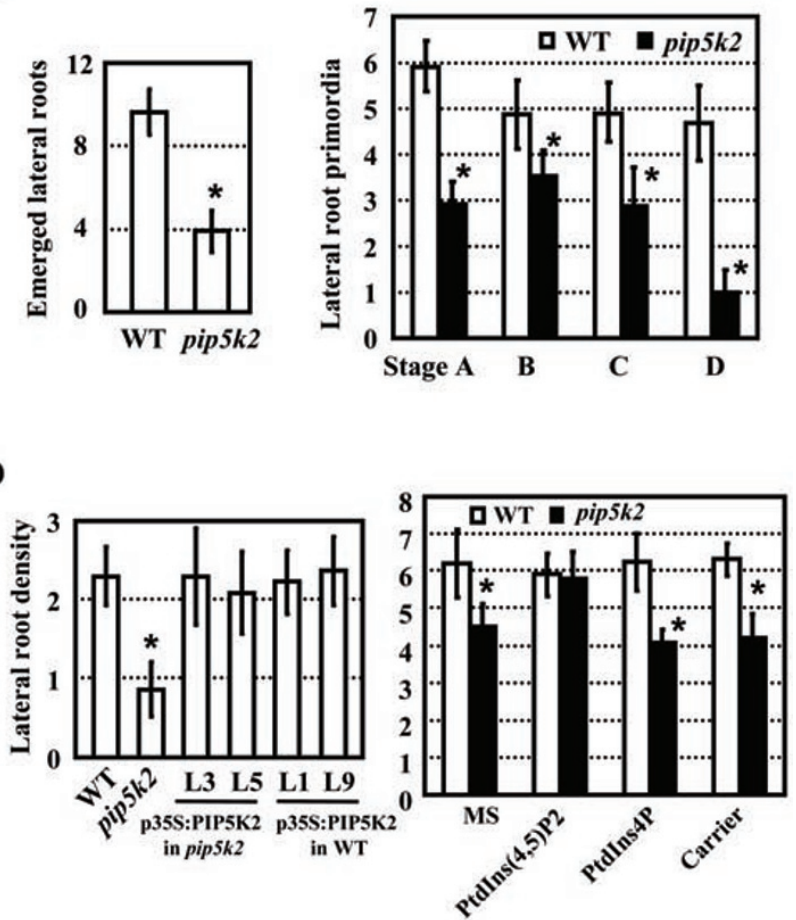

Figure 2 Identification and phenotypic analysis of PIP5K2 knockout mutant pip5k2. (A) Schematic map of the PIP5K2 gene. Exons (brown boxes), introns (lines) and untranslated regions (blue boxes) are indicated. The T-DNA fragment is located at the 1st intron (upper panel). qRT-PCR analysis confirmed the deficiency, overexpression and complemented expression of PIP5K2 in the homozygous pip5k2 mutant plants, WT or pip5k2 plants transformed with p35S:PIP5K2, respectively. L3 and L5 are two independent transgenic pip5k2 lines with complemented expression of PIP5K2, while L1 and L9 are two independent WT lines overexpressing PIP5K2 (same for Figures 2D, 4A, 4C, and 5A). The ACTIN7 gene was used as an internal positive control and the expression of PIP5K2 in WT was set as 1.0 (lower panel). Error bars represent SD $(n=3)$. (B) Phenotypic analysis of 12-day-old pip5k2 seedlings. Reduced lateral root formation is observed and enlarged in the right panel. Scale bar $=1 \mathrm{~cm}$. (C) Measurement and statistical analysis of the number of emerged lateral roots (left panel) and lateral root primordia at different developmental stages (right panel) of WT and pip5k2 seedlings. Twelve-day-old seedlings were measured and statistically analyzed using a heteroscedastic student's $t$-test $\left({ }^{*} P<0.01\right)$. Error bars represent SE $(n>20)$. (D) Lateral root density (formed lateral roots of per centimeter primary root) of 12-day-old WT, pip5k2, transgenic WT or pip $5 k 2$ seedlings with enhanced or rescued expression of PIP5K2 (left panel), and lateral root density of WT and pip5k2 seedlings cultured in liquid MS medium under supplement of phosphatidylinositides (Ptdlns(4,5)P2 or Ptdlns4P) for 9 days (right panel). Statistical analysis was performed using a heteroscedastic Student's $t$-test $\left({ }^{*} P<0.01\right)$. Error bars represent SE $(n>30)$. 
individual transgenic lines were confirmed by qRT-PCR (Figure 2A, lower panel) and measurements of lateral root density revealed that the phenotype of reduced lateral root formation was recovered with transformation rescue of PIP5K2 (Figure 2D, left panel).

To gain further insight into PIP5K2 function, transgenic plants overexpressing PIP5K2 compared to the WT background were also generated. Analysis with qRTPCR confirmed a 3- to 4-fold increase of PIP5K2 expression in individual positive transgenic plants (Figure 2A, lower panel). However, phenotypic observations and measurements showed that the transgenic lines overexpressing PIP5K2 were apparently normal, and no difference in lateral root formation was observed (Figure 2D, left panel).

Considering that PtdIns $(4,5) \mathrm{P}_{2}$ is the direct and major product of PIP5K2, we also tested whether exogenous PtdIns $(4,5) \mathrm{P}_{2}$ could rescue the defect of pip $5 k 2$ mutant. As shown in Figure 2D (right panel), when supplemented with exogenous PtdIns $(4,5) \mathrm{P}_{2}$, the pip $5 k 2$ seedlings could produce as many lateral roots as WT seedlings, while treatment with PtdIns4P or carrier only (which is used to transfer the lipid into plant cells $[22,54])$ had no effect. This indicates that the reduced lateral root formation of pip $5 k 2$ is indeed caused by insufficient endogenous PtdIns $(4,5) \mathrm{P}_{2}$ production.

\section{Auxin accumulation is reduced in pip $5 k 2$}

Much experimental evidence strongly supports a key role of auxin during lateral root formation [55], and application of exogenous auxin results in the induced lateral root formation in Arabidopsis [28]. We thus examined whether exogenous auxin can rescue the lateral root defect in the pip $5 k 2$ mutant. Four-day-old WT and pip $5 k 2$ seedlings (neither of which has lateral roots at this stage) were transferred to medium containing various concentrations of IAA or NAA for eight days, and calculation of the numbers of lateral roots per centimeter of primary root showed that, although the untreated pip $5 \mathrm{k} 2$ seedlings exhibited $\sim 50 \%$ fewer lateral roots, treatment with exogenous IAA or NAA resulted in a similar density of lateral roots as the WT. Treatment with a higher concentration of IAA or NAA resulted in an increased but equivalent number of lateral roots formed in both WT and pip $5 \mathrm{k} 2$ (Figure 3A), demonstrating that exogenous auxin could rescue the lateral root formation phenotype in pip $5 \mathrm{k} 2$.

The regulation of auxin in lateral root development is executed on several different levels. Interruption in auxin accumulation, transport or signaling results in reduced lateral root formation. However, the observation that exogenous IAA recovered the lateral root formation in pip $5 \mathrm{k} 2$ strongly indicates a potential alteration in auxin accumulation or distribution in pip $5 \mathrm{k} 2$ seedlings. To confirm this, Arabidopsis seedlings containing the DR5-GUS cassette (the GUS-coding sequence under transcriptional control of a synthetic auxin-inducible promoter), which has been used widely to detect the auxin accumulation and distribution [56], were included in the study. The DR5-GUS cassette was transferred into pip5k2 through genetic crossing. As shown in Figure 3B, strong GUS signals were detected in the hypocotyl-root junctions and root tips of the WT seedlings; however, GUS signals in pip $5 \mathrm{k} 2$ seedlings were significantly suppressed throughout. In addition, application of exogenous auxin (IAA or NAA, $0.1 \mu \mathrm{M}$ ) resulted in an induction of GUS signals in the roots of both WT and pip $5 k 2$.

Further examination of auxin accumulation in lateral root primordia and emerged lateral roots showed that the GUS signal distribution was not affected in the pip $5 k 2$ seedlings; however, the signal levels were significantly reduced in all developmental stages compared to WT (Figure 3C, left panel). These observations indicate a decrease of free auxin level in pip5k2 seedlings. Considering that DR5-GUS could also reflect the change of auxin response in addition to auxin accumulation and distribution, 10-day-old WT and pip $5 k 2$ seedlings were used to measure the free IAA content, and results showed that the free IAA level in pip $5 \mathrm{k} 2$ was indeed decreased $(\sim 77 \%$ of that in WT, Figure $3 \mathrm{C}$, right panel). These results reveal that auxin accumulation is suppressed under PIP5K2 deficiency, and suggest that the reduction of auxin in lateral root primordia might contribute to the suppressed lateral root initiation.

pip5k2 has delayed root gravity response and is sensitive to the polar auxin transport inhibitor N-1-naphthylphthalamic acid (NPA)

The above observations demonstrate that PIP5K2 deficiency results in reduced lateral root formation which is mainly caused by reduced auxin accumulation, because exogenous IAA could effectively rescue this defect. However, it is still interesting to investigate whether PIP5K2 also has impact on other auxin-related processes. Considering that other two important enzymes in the PI signaling pathway, phospholipase D (PLD) and inositol polyphosphate 5-phosphatase (5PTase) are both involved in auxin transport regulation, the root gravity response - another process regulated by auxin, specifically by polar auxin transport [57-59] was examined. As shown in Figure 4A, measurements of root curvature in response to gravity demonstrated that pip $5 k 2$ bent more slowly than WT, while transgenic lines with transformation rescue of PIP5K2 exhibited similar root curvature as WT, indicating a delayed root gravity response under PIP5K2 
A

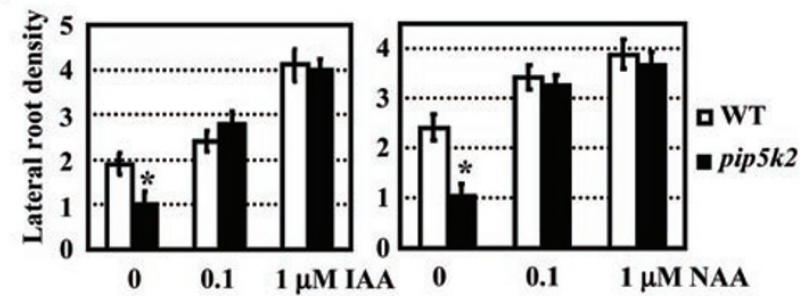

B

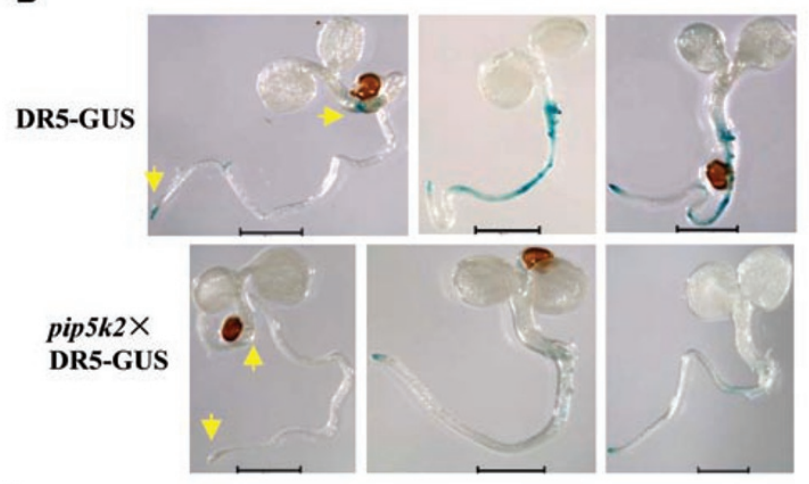

C

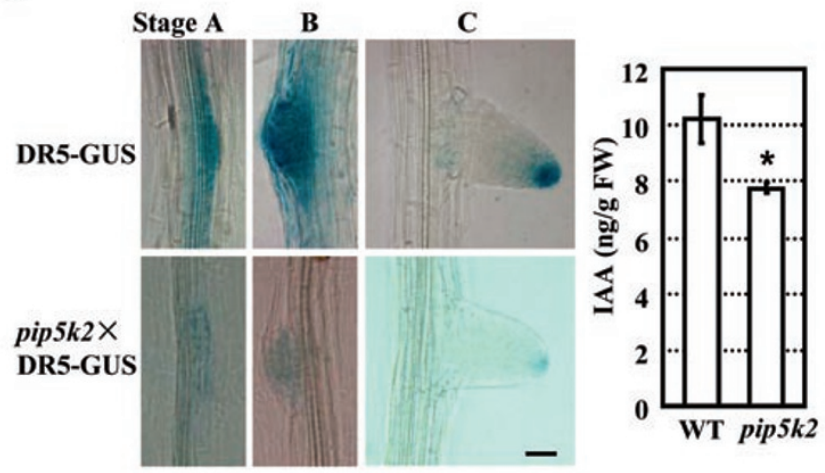

deficiency. As exogenous auxin could recover the reduced lateral root formation and as auxin content is also crucial in gravitropism, the effect of exogenous auxin was examined. After $90^{\circ}$ reorientation for $6 \mathrm{~h}$ and $12 \mathrm{~h}$, the root curvatures of WT and pip $5 k 2$ seedlings treated with IAA or NAA (1 nM or $10 \mathrm{nM})$ were measured and statistically analyzed. The results indicate that treatment with neither IAA nor NAA recovers the reduced root curvature in pip5k2. Treatment with $1 \mathrm{nM}$ IAA resulted in a slight increase, whereas 10 nM IAA caused a $30 \%$ decrease of root curvature in both WT and pip $5 \mathrm{k} 2$ seedlings (Figure 4B, left panel). Similar situation was observed when NAA was applied (Figure 4B, right panel). These findings indicate that the delayed root gravity response in pip $5 \mathrm{k} 2$ is not caused by decreased auxin accumulation, and that the PIP5K2 regulation of root gravity response may be achieved by other routes. A likely possibility is that PIP5K2 regulates root gravity response by affecting
Figure 3 PIP5K2 deficiency results in decreased auxin accumulation. (A) Exogenous IAA (left panel) or NAA (right panel) recovered the reduced lateral root formation of pip5k2. Fourday-old WT and pip5k2 seedlings were transferred to MS medium supplemented with IAA or NAA $(0,0.1$ or $1 \mu \mathrm{M})$ for another 8 days, and lateral root density was measured and statistically analyzed using a heteroscedastic Student's $t$-test $\left({ }^{*} P<0.01\right)$. Error bars represent SE $(n>30)$. (B) Auxin accumulation was decreased in pip5k2 seedlings. GUS expression was detected in the DR5-GUS marker line and pip5k2×DR5-GUS seedlings grown for 6 days after germination (DAG) on MS medium with or without exogenous auxin (IAA or NAA, $0.1 \mu \mathrm{M})$. Arrows highlight the accumulation of auxin at the hypocotyl-root junctions and root tips. Exogenous auxin in low concentrations enhanced the auxin levels in both DR5-GUS and pip5k2×DR5-GUS seedlings. For each treatment, at least 20 seedlings were analyzed and representative images are shown. Scale bar $=1 \mathrm{~mm}$. (C) Significantly reduced auxin accumulation in lateral root primordia and emerged lateral roots of pip $5 k 2$ (left panel). Strong GUS activities were detected in lateral root primordia and emerged lateral roots at different developmental stages of DR5-GUS seedlings, while much weaker GUS signals were detected in pip5k2×DR5-GUS seedlings. A total of 20-30 seedling roots were analyzed and representative images are shown. Scale bar $=20 \mu \mathrm{m}$. Quantitative measurement of free IAA content in 10-day-old WT and pip5k2 seedlings confirmed the decreased IAA amounts (right panel). The measurements were repeated three times and statistically analyzed using a heteroscedastic student's $t$-test $\left({ }^{\star} P<0.01\right)$. Error bars represent SE.

polar auxin transport.

Accordingly, the auxin transport inhibitor-NPA was used to investigate whether polar auxin transport is impaired in pip $5 k 2$. Indeed, the results showed that when seedlings were grown on media containing 0.5 or $1 \mu \mathrm{M}$ NPA, the growth of primary roots was much more inhibited in pip $5 k 2$ than in WT seedlings (Figure 4C, left panel). Further analysis confirmed that pip $5 k 2$ is more sensitive to NPA than WT (Figure 4C, right panel). Not surprisingly, the altered response to NPA by pip $5 \mathrm{k} 2$ was recovered in transgenic lines with transformation rescue of PIP5K2 (Figure 4C, right panel).

Vesicle trafficking is decelerated in pip5k2, which can be recovered by exogenous PtdIns $(4,5) P_{2}$

Research in animals has revealed the critical role of PIP5K and its product PtdIns $(4,5) \mathrm{P}_{2}$ in regulating vesicle trafficking, including both endocytosis and exocytosis. PtdIns $(4,5) \mathrm{P}_{2}$ is able to bind all known endocytic clathrin adapters, and sequestration of $\operatorname{PtdIns}(4,5) \mathrm{P}_{2}$ dominantly inhibits clathrin-mediated endocytosis in vitro $[7,60]$. On the other hand, auxin efflux carriers, PIN1 and PIN2, cycle between the plasma membrane and endosomal compartments, and this cycling is dependent on clathrin- 
mediated endocytosis and intracellular vesicle trafficking $[44,45]$. These clues strongly suggest that PIP5K2 might regulate auxin transport by affecting vesicle trafficking. To test this, FM4-64, a water-soluble and membraneselective fluorescent dye frequently used as a membrane marker to monitor endocytosis in mammalian, fungal and plant cells [61], was used to investigate the potential involvement of PIP5K2 in vesicle trafficking. As shown in Figure 5A, after staining for $30 \mathrm{~min}$, the FM4-64 dye was internalized, and a substantial number of fluorescent vesicles were detected in the cytosol of WT root cells. In contrast, only a few fluorescent vesicles were observed in pip $5 k 2$ root cells, suggesting a severe reduction of the internalization of FM4-64 in pip5k2. To test whether this is caused by PtdIns $(4,5) \mathrm{P}_{2}$ insufficiency, pip $5 k 2$ seedlings pretreated with exogenous $\operatorname{PtdIns}(4,5) \mathrm{P}_{2}$, PtdIns $4 \mathrm{P}$ or the carrier only were examined and an effective recovery of FM4-64 internalization was observed under the treatment of PtdIns $(4,5) \mathrm{P}_{2}$, but not PtdIns4P or the carrier, highlighting the importance of $\operatorname{PtdIns}(4,5) \mathrm{P}_{2}$ in this process. In addition, the reduced vesicle trafficking was also recovered with the transformation rescue of PIP5K2. Together, these results suggest that PIP5K2 positively regulates vesicle trafficking in Arabidopsis root cells, which is probably achieved by its product $\operatorname{Ptd} \operatorname{Ins}(4,5) \mathrm{P}_{2}$.

To confirm whether PtdIns $(4,5) \mathrm{P}_{2}$ has a direct effect on vesicle trafficking, the internalization of FM4-64 was detected in WT root cells pretreated with exogenous

Figure 4 pip $5 k 2$ has delayed root gravity response and is sensitive to polar auxin transport inhibitor NPA. (A) Time course of curvature in seedlings' gravity response tests. Root curvatures of 4-day-old WT, pip5k2, transgenic WT with enhanced PIP5K2 expression, or pip5k2 seedlings with transformation rescue of PIP5K2 expression were measured at different time points after $90^{\circ}$ reorientation. Statistical analysis was performed using a heteroscedastic Student's $t$ -test ( $\left.{ }^{*} P<0.01\right)$. Error bars represent SE $(n>40)$. (B) Exogenous IAA or NAA did not recover the root gravity response of pip $5 \mathrm{k} 2$. Four-day-old WT or pip $5 k 2$ seedlings were vertically grown on MS medium containing 0,1 or $10 \mathrm{nM}$ IAA or NAA and reoriented by $90^{\circ}$ for 6 or $12 \mathrm{~h}$. Root curvatures were measured and statistically analyzed using a heteroscedastic Student's $t$-test, which indicated significant differences compared to WT in all tests $(P<0.01)$. Error bars represent SE $(n>40)$. (C) pip5k2 seedlings were more sensitive to the polar auxin transport inhibitor NPA. Growth of 9-day-old pip5k2 seedlings was inhibited more severely by NPA treatment compared to WT seedlings (left panel). Measurement and calculation of relative root lengths of WT, pip $5 k 2$ and transgenic WT with enhanced PIP5K2 expression, or pip5k2 seedlings with transformation rescue of PIP5K2 expression in the presence of various concentrations of NPA confirm these observations (right panel). Primary root lengths of untreated plants were set as $100 \%$, and statistical analysis was performed using a heteroscedastic Student's $t$-test $\left({ }^{*} P<0.01\right)$. Error bars indicate SE $(n>40)$.
PtdIns $(4,5) \mathrm{P}_{2}$. As shown in Figure 5B, after staining for $10 \mathrm{~min}$, the internalization of FM4-64 was obviously observed in cells pretreated with $\operatorname{PtdIns}(4,5) \mathrm{P}_{2}$, but almost undetectable in controls (treatment with water, PtdIns4P or the carrier, Figure 5B, upper panels). The internalization of FM4-64 could be observed in cells with all kinds of treatment after staining for $30 \mathrm{~min}$ and there are more visible vesicles in $\operatorname{PtdIns}(4,5) \mathrm{P}_{2}$-pretreated cells (Figure 5B, bottom panels). These results confirm that PtdIns $(4,5) \mathrm{P}_{2}$ indeed has a positive effect on vesicle trafficking.

In addition, examination of the sensitivity of pip $5 k 2$

\section{A}

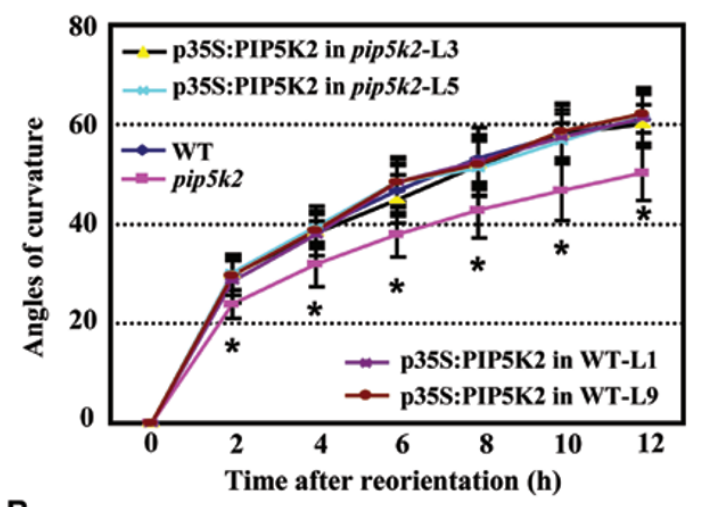

B
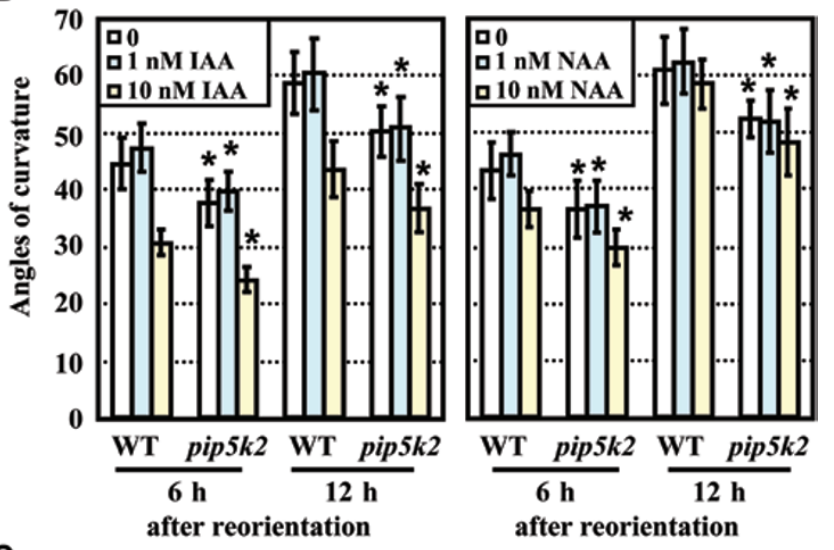

C
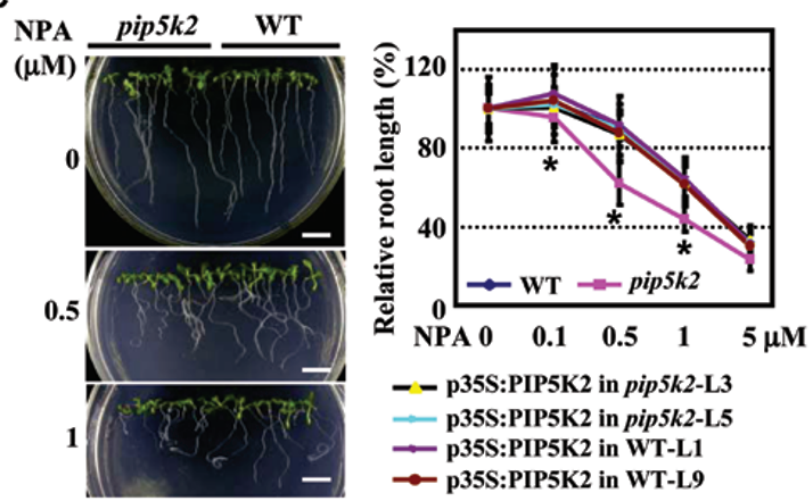
to a vesicle transport inhibitor, BFA, which can inhibit exocytosis to form BFA compartments [62], showed that treatment with $50 \mu \mathrm{M}$ BFA for 30 min inhibited the vesicle trafficking in pip $5 k 2$ root cells, whereas vesicle trafficking was not totally inhibited in WT cells, which had a substantial number of small and dispersed vesicles under the same treatment (Figure 5C, upper panel). Treatment with $100 \mu \mathrm{M}$ BFA resulted in the formation of BFA compartments in both WT and pip $5 k 2$ cells; however, the sizes of the compartments in pip $5 k 2$ cells were much larger (Figure 5C, lower panel), suggesting a more severe inhibitory effect of BFA under PIP $5 K 2$ deficiency.

Due to its inhibitory effect on vesicle trafficking in cells, BFA imposes specific effects on auxin transport-

related processes such as inhibition of root and hypocotyl elongation [43, 46]. Measurement of root lengths of light-grown seedlings and hypocotyl lengths of darkgrown seedlings on MS media containing various concentrations of BFA showed that the inhibition of both primary root and hypocotyl elongation was more pronounced in pip5k2 than in WT (Figure 5D). These observations confirm that pip $5 \mathrm{k} 2$ is more sensitive to BFA, and that vesicle trafficking is indeed suppressed in pip $5 \mathrm{k} 2$ to some extent.

\section{PIP5K2 deficiency caused decelerated polar auxin trans-} port and PIN protein cycling

The delayed root gravity response and hypersensi-

\section{C}

A

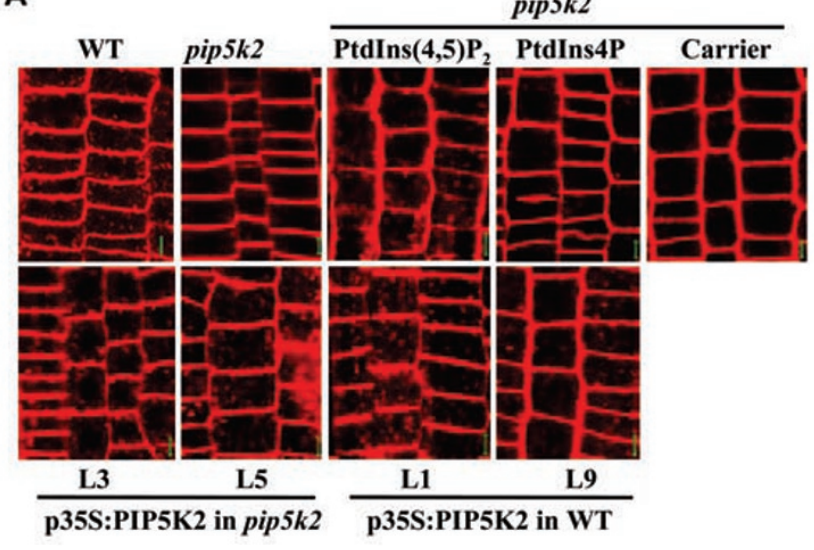

B
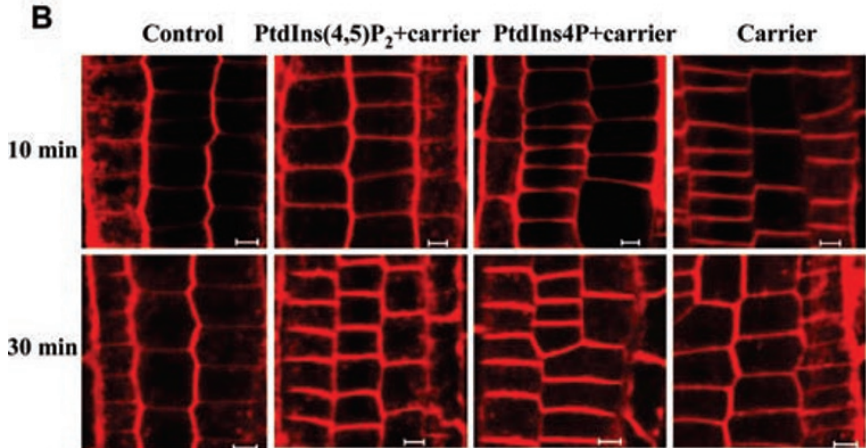

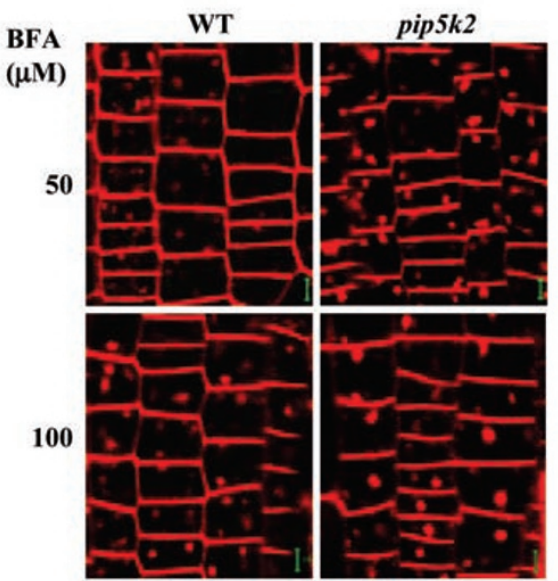

D
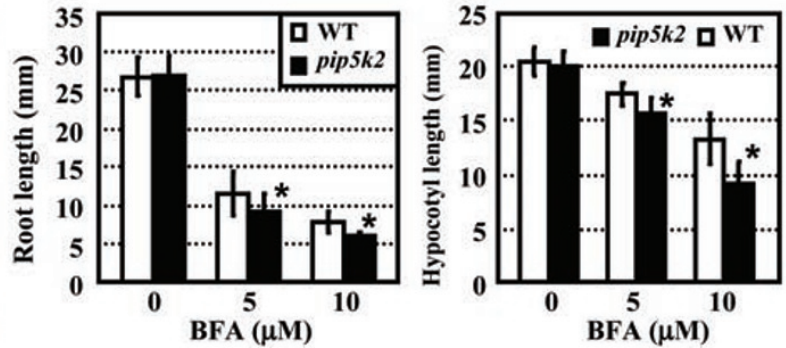

Figure 5 PIP5K2 deficiency caused decelerated vesicle trafficking and hypersensitive responses to BFA. (A) Suppressed vesicle trafficking under PIP5K2 deficiency. Compared to WT, the internalization of FM4-64 was severely reduced in pip5k2, which was recovered by pretreatment with exogenous Ptdlns $(4,5) P_{2}(10 \mu \mathrm{M}, 1 \mathrm{~h})$, but not by Ptdlns $4 \mathrm{P}(10 \mu \mathrm{M}, 1 \mathrm{~h})$ or carrier treatment. FM4-64 internalization in WT overexpressing PIP5K2 or pip5k2 with transformation rescue of PIP5K2 was indistinguishable from that of WT seedlings. Roots of 4-day-old seedlings $(n>20)$ stained with FM4-64 $(5 \mu \mathrm{M}, 30 \mathrm{~min})$ were observed under a confocal microscope. Scale bar $=5 \mu \mathrm{m}$. (B) Vesicle trafficking was accelerated by exogenous $\operatorname{Ptdlns}(4,5) \mathrm{P}_{2}$. Four-day-old WT seedlings $(n>20)$ pretreated with Ptdlns $(4,5) \mathrm{P}_{2}(10 \mu \mathrm{M}, 1 \mathrm{~h})$, Ptdlns $4 \mathrm{P}(10 \mu \mathrm{M}, 1 \mathrm{~h})$ or carrier alone were stained with FM4-64 $(5 \mu \mathrm{M})$ for 10 or 30 min and fluorescence was observed under a confocal microscope. Scale bar $=5 \mu \mathrm{m}$. (C) Formation of BFA compartments was stimulated in pip5k2. Roots of 4-day-old seedlings $(n>20)$ treated with FM4-64 $(5 \mu \mathrm{M})$ and BFA (50 or $100 \mu \mathrm{M}$ ) for 30 min were observed under a confocal microscope. Scale bar $=5 \mu \mathrm{m}$. (D) The pip5k2 seedlings were more sensitive to the inhibitory effects of BFA on root and hypocotyl growth. Root lengths of 7-day-old light-grown seedlings and hypocotyl lengths of 7-day-old dark-grown seedlings on MS medium containing various concentrations of BFA $(0,5,10$ $\mu \mathrm{M})$ were measured and statistically analyzed using a heteroscedastic Student's $t$-test $\left({ }^{*} P<0.01\right)$. Error bars indicate SE $(n>30)$. 
A

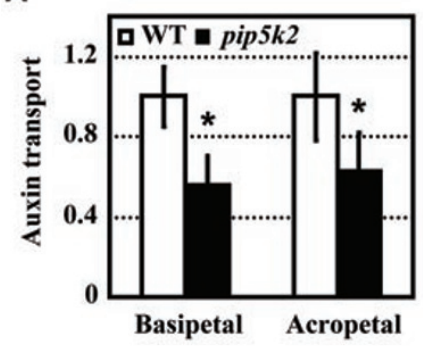

C

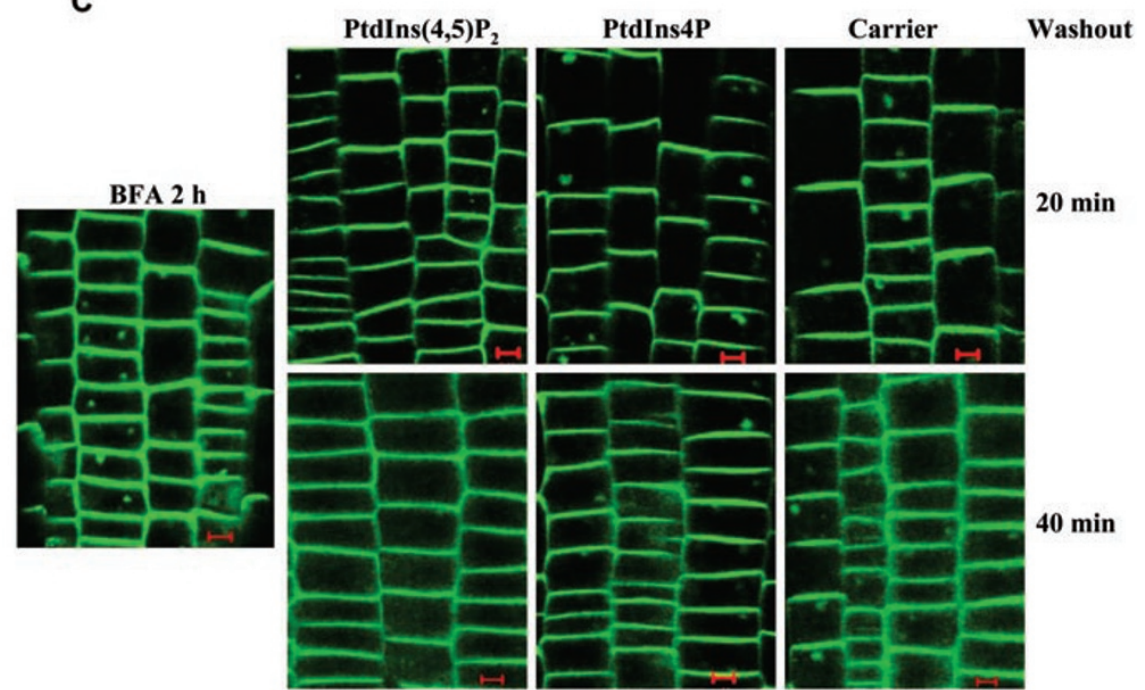

B

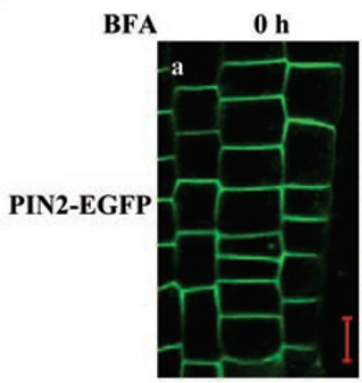

$0.5 \mathrm{~h}$

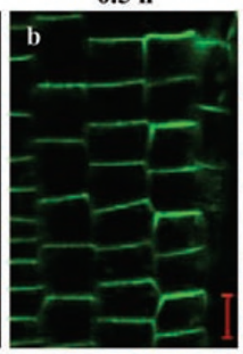

$1 \mathbf{h}$

2 h
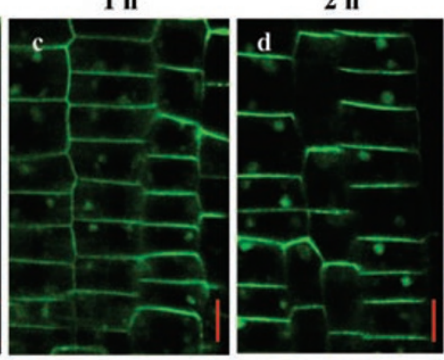

washout $1 \mathrm{~h}$

washout $2 \mathrm{~h}$
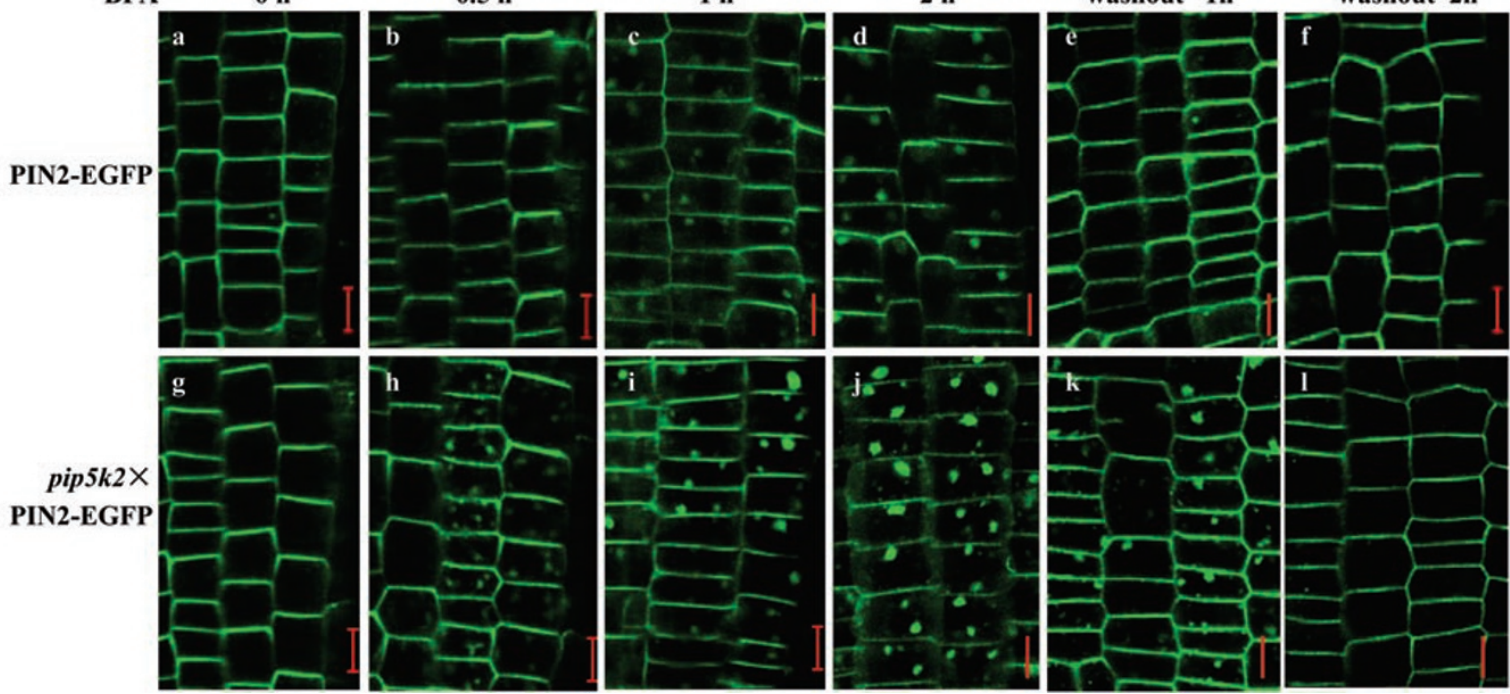

Figure 6 Polar auxin transport and PIN2 cycling are decelerated in pip5k2. (A) Polar auxin transport was decreased in pip5k2. Both acropetal and basipetal auxin transport were detected in the roots of 6-day-old WT and pip5k2 seedlings using $\left[{ }^{3} \mathrm{H}\right]$-labeled IAA. Statistical analysis was performed using a heteroscedastic Student's $t$-test $\left({ }^{\star} P<0.01\right)$. The measurement was repeated three times and error bars represent SE. (B) PIP5K2 deficiency resulted in decelerated PIN2 cycling. Observations of fluorescence by the PIN2-EGFP fusion protein in 4-day-old root cells of WT (a-d) and pip5k2 (g-j) seedlings after treatment with BFA $(50 \mu \mathrm{M})$ for $0,0.5,1$ or $2 \mathrm{~h}$ revealed the enhanced inhibitory effect of BFA on PIN2 cycling in pip5k2×PIN2-EGFP cells. In addition, the recovery of PIN2-EGFP localization after BFA washout for 1 or $2 \mathrm{~h}$ was evidently decelerated under PIP5K2 deficiency $(\mathrm{k}, \mathrm{l})$ compared to WT root cells $(\mathrm{e}, \mathrm{f})$. Scale bar $=10 \mu \mathrm{m}$. (C) Exogenous Ptdlns $(4,5)$ $\mathrm{P}_{2}$ could impair the suppression of PIN2 cycling by BFA. Four-day-old seedlings of PIN2-EGFP were pretreated with BFA (50 $\mu \mathrm{M})$ for $2 \mathrm{~h}$ and then washed with Ptdlns $(4,5) \mathrm{P}_{2}(10 \mu \mathrm{M})$, Ptdlns4P $(10 \mu \mathrm{M})$ or carrier alone for 20 or $40 \mathrm{~min}$. Scale bar $=5 \mu \mathrm{m}$.

tive response to the polar auxin transport inhibitor NPA of pip $5 \mathrm{k} 2$ seedlings suggest that polar auxin transport may be disrupted under PIP5K2 deficiency. To confirm this, 6-day-old WT and pip5k2 seedlings were used for quantitative analysis of polar auxin transport in roots and the results showed that the basipetal auxin transport was reduced to approximately half of that of WT in pip $5 \mathrm{k} 2$ seedlings. In addition, the acropetal auxin transport was also inhibited to $\sim 60 \%$ compared to WT seedlings (Figure 6A), confirming that PIP5K2 deficiency resulted in the reduced polar auxin transport in roots. Although in pin1 seedlings, the decrease in rootward transport is only 
$\sim 30 \%$ under both dark and light conditions [63], we believe that this difference may be due to the differences in plant growth conditions and experimental procedures.

The auxin efflux carrier PIN2 mediates basipetal auxin flux and plays a critical role in root gravity response [37]. Thus, the effects of PIP5K2 on the cycling of PIN2 between the plasma membrane and endosomal compartments were examined. To facilitate PIN2 observation, the PIN2-enhanced green fluorescent protein (EGFP) expression cassette was put into the pip $5 \mathrm{k} 2$ background through genetic crossing. Confocal microscopy observations showed that the polar localization of PIN2 was not affected in pip $5 k 2$ (Figure 6B, a, g). BFA treatment for $0.5 \mathrm{~h}$ had no obvious effect on PIN2-EGFP cycling in control seedlings, whereas a significant aggregation of PIN2-EGFP in the BFA compartments was observed in pip $5 \mathrm{k} 2$ cells (Figure $6 \mathrm{~B}, \mathrm{~b}, \mathrm{~h}$ ). When the treatment time was prolonged to 1 or $2 \mathrm{~h}$, much more PIN2-EGFP aggregates and larger BFA compartments were observed in pip $5 k 2$ cells compared to WT seedlings (Figure 6B, c, d, i, j). Furthermore, after BFA was washed out for $1 \mathrm{~h}$, most BFA compartments disappeared and normal polar localization of PIN2-EGFP was detected in WT (Figure 6B, e). In contrast, accumulated PIN2-EGFP in pip5k2 was not recovered effectively (Figure 6B, k). When BFA was washed out for $2 \mathrm{~h}$, total recovery of PIN2-EGFP localization in both WT and pip $5 k 2$ seedlings was observed (Figure 6B, f, 1). These results indicate that PIN2 cycling between plasma membrane and endosomal compartments is decelerated in pip $5 k 2$.
In order to confirm the effect of PtdIns $(4,5) \mathrm{P}_{2}$ on PIN2 cycling, BFA-pretreated PIN2-EGFP seedlings were washed with buffer containing exogenous $\operatorname{PtdIns}(4,5) \mathrm{P}_{2}$. As shown in Figure $6 \mathrm{C}$, after washing with PtdIns $(4,5)$ $\mathrm{P}_{2}$ for $20 \mathrm{~min}, \sim 80 \%$ of accumulated PIN2-EGFP disappeared, whereas no difference was detected when washing with PtdIns4P or carrier. The localization of PIN2EGFP almost totally recovered when $\operatorname{PtdIns}(4,5) \mathrm{P}_{2}$ washing was extended to $40 \mathrm{~min}$, while clear PIN2-EGFP aggregation can still be observed by washing with PtdIns4P or carrier. This indicates that $\operatorname{PtdIns}(4,5) \mathrm{P}_{2}$ could ameliorate the suppression of PIN2 cycling by BFA. Although PIP5K2/PtdIns $(4,5) \mathrm{P}_{2}$ promotes endocytosis and recycling, the processes are not completely inhibited in pip $5 k 2$ mutant. As BFA mainly blocks exocytosis, the increased BFA bodies can still be observed in pip $5 k 2$ mutant (Figure 6B).

Similarly, PIN3 exhibits a constitutive intracellular cycling between plasma membrane and endosomal compartments as PIN2 does [39]. To further investigate the effect of PIP5K2 on PIN protein cycling, a PIN3-GFP marker line was also included to facilitate the observation of PIN3 subcellular localization and intracellular cycling. Interestingly, confocal microscopy observation showed that similar to PIN2, the cycling of PIN3-GFP was also inhibited to some extent in pip5k2 root cells compared to that in WT. As shown in Figure 7, although BFA treatment for 0 and $0.5 \mathrm{~h}$ had no obvious effect on PIN3-GFP cycling in both WT and pip $5 k 2$ cells (Figure $7, \mathrm{a}, \mathrm{b}, \mathrm{g}, \mathrm{h}$ ), BFA treatment for 1 or $2 \mathrm{~h}$ resulted in much
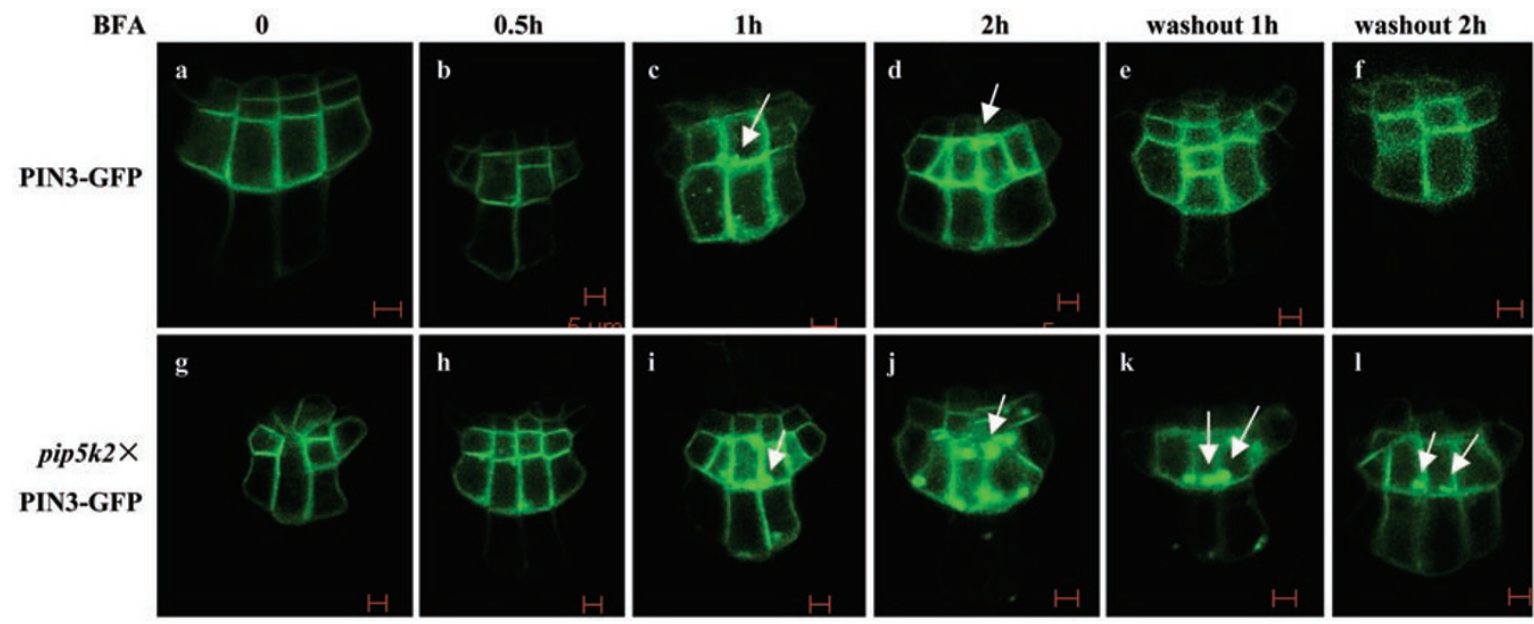

Figure 7 Intracellular cycling of PIN3 is decelerated in pip5k2. PIP5K2 deficiency resulted in decelerated PIN3 cycling. Observations of fluorescence by the PIN3-GFP fusion protein in 4-day-old root cells of WT (a-d) and pip5k2 (g-j) seedlings after treatment with BFA $(50 \mu \mathrm{M})$ for $0,0.5,1$ or $2 \mathrm{~h}$ revealed the enhanced inhibitory effect of BFA on PIN3 cycling in pip5k2×PIN3-GFP cells. In addition, the recovery of PIN3-GFP localization after BFA washout for 1 or $2 \mathrm{~h}$ was evidently decelerated under PIP5K2 deficiency (k, I) compared to WT root cells (e, f). Scale bar $=5 \mu \mathrm{m}$. 
enhanced PIN3-GFP aggregation in the BFA compartments in pip5k2 root cells (Figure 7, c, d, i, j). In addition, washout of BFA for $1 \mathrm{~h}$ was sufficient for recovery of the normal localization of PIN3-GFP in WT (Figure 7, e), while accumulated PIN3-GFP still remained in pip $5 k 2$ cells (Figure 7, k). Even after BFA was washed out for $2 \mathrm{~h}$, small amount of PIN3-GFP aggregation could still be observed in pip $5 k 2$ cells (Figure 7, 1), while in WT cells the effect of BFA was totally eliminated (Figure 7, f). These observations reveal that PIP5K2 is required for both PIN2 and PIN3 cycling and suggest that the regulation of these PIN proteins may contribute to the role of PIP5K2 in root gravity response.

Suppressed redistribution of auxin and PIN2 under gravistimulation in pip $5 k 2$

In agreement with the classic Cholodny-Went theory, recent studies have shown the formation of lateral auxin gradients in Arabidopsis roots upon gravistimulation [6466]. To determine whether the reduced gravity response in pip $5 \mathrm{k} 2$ is accompanied by an alteration in the asymmetric distribution of auxin in the root tip, GUS activity under gravistimulation was analyzed in WT and pip $5 k 2$ seedlings containing the DR5-GUS cassette. It has been reported that the DR5-GFP signal is detected to relocate in the root tip after gravistimulation for 3 hours, and compared with DR5-GFP, as the detection of DR5-GUS need staining with GUS buffer for about 4 hours, thus the relocation of DR5-GUS signal could be observed later after gravitropism [67]. As shown in Figure 8A, after gravistimulation for $4 \mathrm{~h}$, there was no obvious auxin redistribution in WT or pip $5 k 2$ seedlings compared to nonstimulated seedlings. However, when the stimulation was extended to $6 \mathrm{~h}, \sim 50 \%$ of WT seedlings exhibited extended auxin redistribution basipetally along the gravity side, whereas this effect was almost undetectable in pip $5 \mathrm{k} 2$ seedlings. After gravistimulation for $8 \mathrm{~h}$, auxin redistribution was more obvious in WT seedlings, i.e., the area of auxin redistribution is expanded to the curve of the roots, while still no obvious difference was detected in pip $5 k 2$ seedlings compared to nonstimulated ones.

In WT Arabidopsis roots, asymmetric PIN2 distribution can be detected after 1-2 h of gravistimulation and the PIN2-specific signals are weaker at the upper side than the lower side of horizontally positioned roots, especially in epidermal cell layer [68], which is regulated by intracellular vesicle trafficking and could result in gravistimulated auxin redistribution. Considering that pip $5 k 2$ shows both altered PIN2 trafficking and auxin redistribution, microscopy observation of PIN2 distribution under gravistimulation showed that the differential distribution of PIN2 in WT seedlings appeared after 1.5-

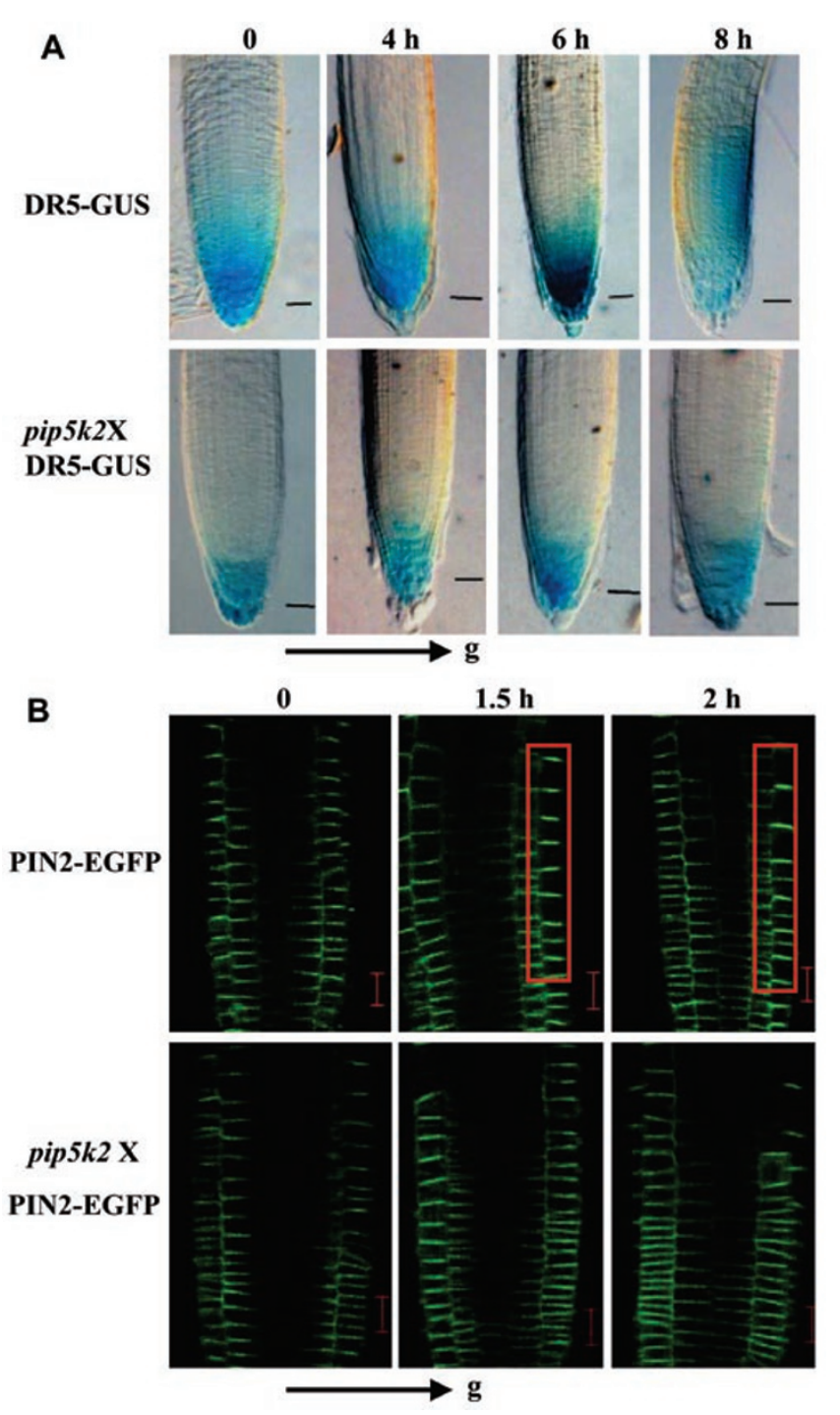

Figure 8 Auxin and PIN2 redistribution under gravistimulation is suppressed in pip5k2. (A) GUS expressions are detected in root tips of DR5-GUS and pip5k2×DR5-GUS seedlings $(n>30)$ before and after $90^{\circ}$ reorientation for 4,6 or $8 \mathrm{~h}$ and representative images are shown. Arrow indicates the direction of gravistimulation. Scale bar $=10 \mu \mathrm{m}$. (B) Observations of fluorescence by the PIN2-EGFP fusion protein in 4-day-old root cells of WT and pip5k2 seedlings before or after $90^{\circ}$ reorientation for 1.5 or $2 \mathrm{~h}$. Asymmetric distribution of PIN2 in WT seedlings is highlighted by red boxes, whereas no differential distribution was observed in pip5k2 seedlings. Scale bar $=10 \mu \mathrm{m}$.

$2 \mathrm{~h}$ of gravistimulation; however, no distinct difference was detected in pip $5 \mathrm{k} 2$ seedlings after $2 \mathrm{~h}$ of gravistimulation (Figure $8 \mathrm{~B}$ ). These observations confirm that both auxin and PIN2 redistribution under gravistimulation are suppressed in pip $5 k 2$, consistent with the reduced gravity response. 


\section{Discussion}

The activity of Arabidopsis PIP5Ks has been examined in either bacteria or insect cells. In roots, the activity of PIP5K2 converting PtdIns4P into PtdIns $(4,5) \mathrm{P}_{2}$ is only lower than PIP5K3 and is much higher than other isoforms [16]. In this study, we demonstrate that PIP5K2 is necessary for lateral root formation and root gravity response by affecting auxin accumulation and PIN-mediated polar auxin transport through regulation of vesicle trafficking.

PIP5K2/PtdIns $(4,5) P_{2}$ regulates vesicle trafficking and is crucial for lateral root formation

Comparing with PIP5K3 that is expressed only in the root epidermal and root hair cells, the PIP5K2 expression is much broader and at higher levels. Thus it is reasonable to speculate that PIP5K2 is the main source of PtdIns $(4,5) \mathrm{P}_{2}$ production in roots. In addition, PIP5K2 shows a preferential subcellular localization at the plasma membrane, forms aggregates in the BFA compartment after BFA treatment, and interacts with RabE GTPases which are involved in anterograde membrane trafficking $[69,70]$, indicating a possible role of PIP5K2 in vesicle trafficking. Our observations confirm that the internalization of the membrane marker FM4-64 is suppressed in pip $5 k 2$; this functionality can be recovered by both exogenous PtdIns $(4,5) \mathrm{P}_{2}$ treatment and complementation by PIP5K2 expression (Figure 5A). In addition, exogenous PtdIns $(4,5) \mathrm{P}_{2}$ stimulates the internalization process in WT cells (Figure 5B), which strongly suggests that vesicle trafficking, especially endocytosis, is affected by PIP5K2 through its product PtdIns(4,5) $\mathrm{P}_{2}$. These results are similar to previous findings that disruption of PIP5K4 inhibits proper endocytosis and membrane recycling in pollen tubes [21] and consistent with the report that PIP5K6 and PtdIns $(4,5) \mathrm{P}_{2}$ positively regulate clathrin-dependent endocytosis [18].

Experiments employing BFA further confirmed the function of PIP5K2 in the regulation of vesicle trafficking and the possible participation of PIP5K2 in an anterograde trafficking process. These agree well with the fact that PIP5K4 and PIP5K5 regulate pollen tube growth through vesicle trafficking and pectin deposition, which is more related to anterograde trafficking and exocytosis, and further reveal that besides in pollen tubes, Arabidopsis PIP5K also regulates vesicle trafficking in root cells. Together with previous studies on Arabidopsis PIP5K3, PIP5K4 and PIP5K5, it is suggested that plant PIP5Ks may have the same function as their animal counterparts in both endocytosis and exocytosis. However, we cannot exclude the possibility that the effect of PIP5K2 could be indirect, through $\operatorname{Ins}(1,4,5) \mathrm{P}_{3}$ and $\operatorname{Ins}(1,4,5) \mathrm{P}_{3}$-induced calcium signaling.

Significant reduction in the number of both lateral root primordia and lateral roots of all the four developmental stages in pip $5 \mathrm{k} 2$ seedlings is consistent with the specific and strong expression of PIP5K2 in lateral root primordia and emerged lateral roots. This is different from the expression of PIP5K1 in procambial tissues of leaves, flowers and roots [15], indicating a crucial role of PIP5K2 in lateral root initiation. Exogenous IAA treatment effectively recovered the lateral root phenotype, confirming the involvement of auxin and strongly suggesting a defect of auxin accumulation or distribution in pip $5 k 2$ seedlings, which is further confirmed by the reduced free IAA content in pip $5 k 2$ seedlings. These results support the hypothesis that PIP5K2 regulates lateral root formation by modulating auxin accumulation in spite of the fact that polar auxin transport and the function of PIN proteins (which are also affected by PIP5K2 deficiency) play important roles in lateral root initiation as well [24, 71]. By qRT-PCR analysis, we found that the expression of auxin biosynthesis genes CYP79B2, CYP83B1 and NIT3 was suppressed (to $\sim 1 / 3$ of the levels in WT seedlings) and the transcription levels of GH3.2 and GH3.5, which are IAA-amino-acid synthase encoding genes, were stimulated in pip $5 k 2$ (Supplementary information, Figure S1), which could be the possible mechanism of how PIP5K2 affects auxin accumulation. Thus, expression of both auxin biosynthesis and metabolism genes was modified, reflecting a bidirectional regulation by PIP5K2 on auxin accumulation.

Application of exogenous PtdIns $(4,5) \mathrm{P}_{2}$ rescues the phenotype of pip $5 \mathrm{k} 2$ and confirms the importance of PtdIns $(4,5) \mathrm{P}_{2}$ in the lateral root formation and root gravity response; however, the PIP5K2-overexpression seedlings did not show any phenotypic difference from WT ones. This may be because the activity of PIP5K is regulated by post-transcriptional modifications, such as phosphorylation or de-phosphorylation, or that the product $\operatorname{PtdIns}(4,5) \mathrm{P}_{2}$ is involved in multiple processes, and the presence of complicated regulation routes may balance the elevation of PtdIns $(4,5) \mathrm{P}_{2}$ levels in plant cells. As $\operatorname{Ins}(1,4,5) \mathrm{P}_{3}$, the direct product of $\operatorname{PtdIns}(4,5) \mathrm{P}_{2}$, plays a critical role in the early phase of gravistimulation [40], it is also possible that the elevation of PIP5K2 expression does not result in the ultimate elevation of in vivo Ins $(1,4,5) \mathrm{P}_{3}$ level, and hence no difference in gravity response was observed for PIP5K2-overexpression lines.

The regulatory role of PIP5K2 in root gravity response is likely achieved by modulating PIN protein cycling

The delayed root gravity response, which could not be 
recovered by exogenous IAA and NAA, the hypersensitive response to the polar auxin transport inhibitor NPA and the reduced basipetal and acropetal auxin transport indicate that PIP5K2 is involved in the regulation of polar auxin transport.

PIN2, a well-characterized auxin efflux facilitator, mediates basipetal auxin transport and is crucial for gravitropic response. Studies show that PIN2 undergoes cycling between membrane and endosomal compartments, which is dependent on the clathrin machinery and vesicle trafficking $[44,45]$. The regulatory role of PIP5K6 and $\operatorname{PtdIns}(4,5) \mathrm{P}_{2}$ in clathrin-dependent endocytosis and decelerated vesicle trafficking (both endocytosis and exocytosis) in pip $5 k 2$ suggest that PIN2 cycling might be affected. This is confirmed by further analysis of PIN2 localization and cycling employing BFA and the recovery of polar localization after removal of BFA (Figure 6B). The positive effect of exogenous $\operatorname{PtdIns}(4,5) \mathrm{P}_{2}$ on the recovery process highlights the importance of $\operatorname{PtdIns}(4,5)$ $\mathrm{P}_{2}$ and PIP5K2 (Figure 6C). Furthermore, PIN3, which is also a critical factor in gravitropic response, was similarly affected as PIN2 in pip5k2 seedlings (Figure 7). This might ultimately result in a delayed root gravity response through interference with basipetal auxin transport. A similar extent of reduction in basipetal auxin transport was detected in pip $5 k 2$ compared to pin 2 mutant, which shows stronger defects in root gravity response. This may be due to the different measurement protocols, such as the different incubation time with $\left[{ }^{3} \mathrm{H}\right]$-IAA and sections used for radioactivity detection. Inhibited asymmetric PIN2 localization and auxin redistribution under gravistimulation in pip $5 \mathrm{k} 2$ seedlings (Figure 8 ) correlates well with the reduced gravity response, and supports the idea that PIN2 is at least one important downstream factor mediating the regulatory function of PIP5K2 in polar auxin transport and gravity response, thus implicating a potential effect of PIP5K2 on early phases of gravity signal transduction.

Besides the fact that PIP5K2 regulates the PIN protein cycling through PtdIns(4,5) $\mathrm{P}_{2}$-mediated vesicle trafficking process, another possibility is that PIP5K 2 deficiency and consequent insufficiency of $\operatorname{PtdIns}(4,5) \mathrm{P}_{2}$ may cause changes of the whole-membrane lipid composition, some of which are known to be critical for PIN cycling [47, 48]. Although the possible roles of Arabidopsis PIPKs in vesicle regulation have been indicated [16-18, 20, 22], the downstream targets are not well characterized yet. Our results reveal for the first time that PIP5K2 affects the function of a specific target, PIN proteins, by regulating vesicle trafficking, to participate in root gravity response. This finding also sheds new light on the crosstalk between PI signaling and auxin response, both of which have crucial regulatory roles in plant development.

$\operatorname{PtdIns}(4,5) \mathrm{P}_{2}$ can directly stimulate the assembly of clathrin-coated vesicles, which is important for the cycling of PIN proteins [45]. In addition, both PIP5K and PtdIns $(4,5) \mathrm{P}_{2}$ bind to and regulate the activity of certain small GTPases [72]. Particularly, PIP5K2 itself interacts with RabE GTPases, which provide the energy source for vesicle trafficking inside the cells. It is possible that the regulation of PIP5K2 on PIN proteins is mediated through affecting the clathrin machinery. In addition to PIN proteins, other integral membrane proteins, such as ion channels, pumps and ATPases, could also be targets of PIP5K2/PtdIns $(4,5) \mathrm{P}_{2}$ and the consequent change in membrane composition may also contribute to the reduced polar auxin transport observed in pip $5 \mathrm{k} 2$. Thus, PIP5K2/PtdIns $(4,5) \mathrm{P}_{2}$ likely play diverse roles in multiple cellular processes.

\section{Materials and Methods}

\section{Plant material and growth conditions}

Arabidopsis thaliana ecotype Columbia was used in the study as the plant material. Plant transformation was performed using the floral dip method [73]. Seeds were surface sterilized with $20 \%$ (v/v) bleach and sown on Murashige and Skoog (MS) plates. Oneweek-old seedlings were transferred to soil and grown at $22{ }^{\circ} \mathrm{C}$ under a 16-h light / 8-h dark photoperiod.

\section{Quantitative real-time RT-PCR ( $q R T-P C R)$ analysis}

qRT-PCR analysis was performed to study the transcription levels of PIP5K2 in various Arabidopsis tissues, insertion mutant or transgenic plants, as well as the expression pattern of auxin biosynthesis- and metabolism-related genes. Ten-day-old seedlings of pip $5 k 2$, different transgenic plants, or WT seedlings growing on standard MS plates treated with IAA $(0.1,1$ and $10 \mu \mathrm{M})$ or ICA $(10 \mu \mathrm{M})$ by transferring them into liquid MS media containing corresponding IAA or ICA for $0,0.5,1,2,3,6$ and $12 \mathrm{~h}$, were harvested for RNA extraction. PCR amplification was executed with the RotorGene 3000 system (Corbett Research) using the SYBR green detection protocol (Toyobo, Tokyo, Japan). The Arabidopsis ACTIN7 gene was used as an internal control, and differences in product levels among the tested samples during the linear amplification phase were used to calculate differential gene expressions. The primers used are as follows: PIP5K2 (5'-GAAGAATGAGTTGATTGTTGCGAC-3' and 5'-AGATAGATGCGGTGGTGTGGTC-3'), and ACTIN7 (5'-TTCCCGTTCTGCGGTAGTGG-3' and 5'-CCGGTATTGTGCTCGATTCTG-3').

To analyze the expression of auxin biosynthesis- and metabolism-related genes, 10-day-old WT and pip $5 k 2$ seedlings were harvested for RNA extraction. The genes tested were as follows: TRP2 (5'-TTGAATCCGCTTTCTATGCTCT-3' and 5'-CTGTAATGCTCCGTAAGCCTCT-3'), TRP3 (5'-ATCATCTGTAAGCGGAAAGGTTC-3' and 5'-TTCAGTTGGCGACTTTGCATCAC-3'), CYP79B2 (5'-ATCTGCCGATGCTCACTGGA-3' and 5'-GCCGCCATTACAAGCTCCTTA-3'), YUCCA3 (5'-CGTTCGTAGCGCTGTTCATG-3' and 5'-CTAACGGTCCAATTTTCG- 
GC-3'), AAO1 (5'-CTGGTGAGGCTGTTTATGTAGACG-3' and 5'-CAACCAAAAAGGCGATTATCTGA-3'), AMI1 (5'-TCTACTTCCTCGTCGCCTCCT-3' and 5'-GCGCATTTTCTCCGTTTATACTG-3'), TAA1 (5'-TAAACACTATACAAACGACCAAACC-3' and 5'-TACACCTGTCACCCATCTTCCT-3'), CYP83B1 (5'-ACCCTAACCGCCCTAAACAAGA-3' and 5'-GTCAGTTCCCGGCACAACAATA-3'), NIT3 (5'-AGGTTATTGGCGTTGACCCAT-3' and 5'-ATCTTTCCACTTCAGGGCCAG-3'), GH3.2 (5'-CAAGCTCAGGAACATCTGCTG-3' and 5'-TCGTCTCTTGAAGTGGTCGCT-3'), GH3.5 (5'-AGGCGACACGACCTCAACG-3' and 5'-GGAACGAACTGGCTCATCACA-3') and ILL2 (5'-GGATGCTTTGCCTATTCAGGAA-3' and 5'-GGCAGAAAGATGTATCCCGAAA-3').

Promoter-GUS fusion studies and histochemical analysis of GUS activity

The $\sim 1.9-\mathrm{kb}$ PIP $5 K 2$ promoter region was amplified by PCR using primers PIP5K2p-1 (5'-CCCAAGCTTATCATTACCTCGTGCTCTTCA-3', added HindIII site underlined) and PIP5K2p-2 (5'-CGGGATCCGATTACGGATTAGGGTGA-3', added BamHI site underlined) using Arabidopsis genomic DNA as the template. The amplified DNA fragment was subcloned into pCAMBIA1300+pBI101.1 [74] and the resultant construct was transformed into Arabidopsis. Positive transgenic plants were selected through hygromycin resistance screening, and independent lines of T2 and T3 homozygous progeny were used to detect GUS activity. To determine the auxin distribution, 6-day-old DR5-GUS and pip $5 k 2 \times$ DR5-GUS seedlings were used. Photography was performed using a Nikon microscope (SMZ800) with a digital camera (Nikon, Coolpix 4500).

T-DNA insertion knockout mutant identification, overexpression and transformation rescue studies of PIP5K2

A putative knockout mutant of PIP5K2, SALK_012487, was identified in the Salk Institute T-DNA insertion library database (http://signal.salk.edu/cgi-bin/tdnaexpress; [52]) by searching with locus At1g77740. PCR amplification using primer LBa1 (5'-TGGTTCACGTAGTGGGCCATCG-3') and gene-specific primer PIP5K2-1 (5'-GAAGAATGAGTTGATTGTTGCGAC-3'), or primers PIP5K2-1 and PIP5K2-2 (5'-AGATAGATGCGGTGGTGTGGTC-3'), was performed to confirm the insertion of T-DNA or to identify the homozygous mutant lines.

The entire PIP5K2 cDNA fragment was amplified with primers PIP5K2-3 (5'-AGATGATGCGTGAACCGCTT-3') and PIP5K2-4 (5'-GCTCTAGATTAGCCGTCTTCGATGAAGATTC-3', added SalI site underlined) and subcloned into pCAMBIA1301 to generate p35S:PIP5K2 harboring PIP5K2 in the sense orientation. The construct was transformed into WT and pip $5 k 2$ for overexpression and complementation studies, respectively. The obtained transformants were selected by hygromycin resistance screening. Transcription levels of PIP5K2 in pip5k2 mutant, transgenic WT or pip $5 k 2$ seedlings with enhanced or transformation rescue of PIP5K2 were confirmed by qRT-PCR.

\section{Phenotypic analysis of pip5k2 and transgenic plants}

Roots of 12-day-old light-grown seedlings on vertical plates were cleared and observed under a Leica DMR microscope using a $40 \times$ objective to classify the stage of lateral root development according to Zhang et al. [53]. Lateral root primordia and emerged lateral roots at different stages were quantified and statistically analyzed.

To test the effect of IAA or NAA on lateral root formation, 4-day-old light-grown seedlings were transferred to MS medium containing different concentrations of IAA or NAA $(0,0.1$ and 1 $\mu \mathrm{M})$ for another 8 days, and the number of lateral roots formed per centimeter of primary root was calculated.

The root gravity response was determined by using 4-day-old light-grown seedlings. The angles of root curvature were measured at $2,4,6,8,10$ and $12 \mathrm{~h}$ after $90^{\circ}$ reorientation in the dark, with the aid of the image-analysis program ImageJ (version 1.34 for Windows; http://rsb.info.nih.gov/ij/). To test the effect of exogenous auxin on root gravity response, various concentrations of IAA or NAA were used, and the angle of root curvature after $90^{\circ}$ reorientation was measured after 6 and $12 \mathrm{~h}$ in darkness.

\section{FM4-64 staining and confocal microscopy observation}

Roots of 4-day-old WT, pip $5 k 2$, transgenic WT or pip $5 k 2$ seedlings with enhanced or transformation rescue of PIP5K2 were stained with $5 \mu \mathrm{M}$ FM4-64 (Invitrogen 454218, California, USA) for $10 \mathrm{~min}$, followed by washing with water three times. After incubation in water for $30 \mathrm{~min}$ at room temperature, the fluorescence was observed with a confocal laser-scanning microscope with an excitation wavelength of $543 \mathrm{~nm}$ (Zeiss LSM 510 META). To monitor vesicle trafficking under phospholipids treatment, the seedlings were incubated with $10 \mu \mathrm{M}$ PtdIns $(4,5) \mathrm{P}_{2}$ (SigmaAldrich 64365, Missouri, USA) or $10 \mu \mathrm{M}$ PtdIns4P (Sigma-Aldrich P7686) for $1 \mathrm{~h}$ prior to FM4-64 staining. The phospholipids were delivered into the cells using the polyamine histone H1 PI carrier (Invitrogen 78562) according to the manufacturer's instructions. Treatment of the carrier alone was used as the negative control. All images within a single experiment were captured with the same gain and exposure settings.

\section{NPA and BFA treatments}

The primary root lengths of 9-day-old seedlings grown on MS medium supplemented with auxin transport inhibitor NPA (Duchefa Biochemie N0926, Haarlem, Netherlands) were measured and calculated to test the sensitivity to NPA.

To test sensitivity to the vesicle trafficking inhibitor BFA on the physiological level, sterilized seeds of WT and pip $5 k 2$ were germinated on MS medium supplemented with various concentrations of BFA (Sigma-Aldrich B-7651). The primary root lengths of 7-day-old light-grown seedlings and hypocotyl lengths of 7-dayold dark-grown seedlings were measured and calculated.

\section{Genetic crosses}

Genetic crossing was carried out to transfer the DR5-GUS, PIN2-EGFP and PIN3-GFP cassette individually into pip $5 k 2$ plants. The pip $5 k 2$ plants were used as the female parent, while DR5-GUS, PIN2-EGFP and PIN3-GFP transgenic plants were used as the male parent. Homozygous cross offspring were used for further analysis.

Visualization of BFA compartments, PIN protein cycling and PIN2 distribution under gravistimulation

Roots of 4-day-old WT and pip5k2 seedlings were incubated with a solution containing FM4-64 $(5 \mu \mathrm{M})$ and BFA (50 or 100 $\mu \mathrm{M})$ for $30 \mathrm{~min}$, and then BFA compartments were examined by 
confocal laser scanning microscopy.

To monitor PIN-protein cycling, the PIN2-EGFP or PIN3-GFP cassette was transferred into pip $5 k 2$ by genetic crossing. PINprotein localization in seedlings treated with $50 \mu \mathrm{M}$ BFA for $0,0.5$, 1 and $2 \mathrm{~h}$, or $2 \mathrm{~h}$ treatment with $50 \mu \mathrm{M}$ BFA followed by 1 and 2 $\mathrm{h}$ washing, was observed using confocal laser scanning microscopy with an excitation wavelength of $488 \mathrm{~nm}$. In addition, the BFA- $(50 \mu \mathrm{M}, 2 \mathrm{~h})$ pretreated PIN2-EGFP seedlings washed with PtdIns $(4,5) \mathrm{P}_{2}(10 \mu \mathrm{M})$, PtdIns4P $(10 \mu \mathrm{M})$ or carrier alone for 10 or $30 \mathrm{~min}$ were used to observe the fluorescent signals. To detect the PIN2 distribution under gravistimulation, fluorescent signals of 4-day-old PIN2-EGFP and pip5k2×PIN2-EGFP vertical positioned seedlings were observed before or after $90^{\circ}$ reorientation for 1.5 or $2 \mathrm{~h}$.

\section{Measurement of auxin content and auxin transport in seed- lings}

Ten-day-old WT and pip5k2 seedlings growing under normal conditions were harvested and freeze-dried. Measurement of free IAA content was performed using LC-MS according to Kowalczyk and Sandberg [75].

Auxin transport assay was performed according to previous description [76]. Ten-mm root segments were cut from 6-day-old WT and pip $5 k 2$ seedlings, and aligned root apices were used for measurement of the polar auxin transport. To test the basipetal auxin transport, agar blocks of $1 \mathrm{~mm}$ diameter containing $200 \mathrm{nM}\left[{ }^{3} \mathrm{H}\right]-$ IAA ( $23 \mathrm{Ci} / \mathrm{mmol}$, Amersham) were applied at the root tip. After incubation for $8 \mathrm{~h}, 5-\mathrm{mm}$ sections of the root away from the root tip were collected and placed into scintillation vials containing $2 \mathrm{ml}$ scintillation fluid to examine the radioactivity with a liquid scintillation counter (1450 MicroBeta TriLux; Perkin-Elmer) after $18 \mathrm{~h}$ incubation. For measurement of acropetal auxin transport, agar blocks containing $\left.200 \mathrm{nM} \mathrm{[}{ }^{3} \mathrm{H}\right]$-IAA were placed $10 \mathrm{~mm}$ back from the root tip and incubated for $12 \mathrm{~h}$; after that 5-mm root segments containing the root tip were harvested to test the radioactivity as described above. Thirty seedlings were used for each assay and the measurements were repeated three times.

\section{Accession numbers}

Sequence data generated or used in this study can be found in the EMBL/GenBank database under the following accession numbers: PIP5K2 (At1g77740, NM_106423), ACTIN7 (At5g09810, NM_121018), TRP2 (At5g54810, NM_124862), TRP3 (At3g54640, NM_115321), CYP79B2 (At4g39950, NM_120158), YUCCA3 (At1g04610, NM_100340), AAO1 (At5g20960, NM_122105), AMI (Atlg08980, NM_100769), TAA1 (Atlg70560, NM_105724), CYP83B1 (At4g315̄00, NM_119299), NIT3 (At3̈g44320, NM_114300), GH3.2 (At4g37390, NM_119902), GH3.5 (At4g)27260, NM_118860), ILL2 (At5g56660, NM_125049).

\section{Acknowledgments}

This work was supported by National Natural Science Foundation of China (31130060, 90717001) and Science and Technology Commission of Shanghai Municipality (08XD14049). We thank Dr Jian Xu (Utrecht University) for providing the Arabidopsis transgenic lines harboring DR5-GUS and PIN2-EGFP expression cassettes. We thank Dr Chuan-You Li (Institute of Genetics and Developmental Biology, CAS) for help on measurement of polar auxin transport, and Dr Lang-Tao Xiao (Hunan Agricultural University) for help on measurement of auxin content. We also thank Mr Xiao-Shu Gao for help on confocal microscope observation.

\section{References}

1 Stevenson JM, Perera IY, Heilmann I, Persson S, Boss WF. Inositol signaling and plant growth. Trends Plant Sci 2000; 5:252-258.

2 Xue HW, Chen X, Li G. Involvement of phospholipid signaling in plant growth and hormone effects. Curr Opin Plant Biol 2007; 10:483-489.

3 Xue HW, Chen X, Mei Y. Function and regulation of phospholipid signalling in plants. Biochem J 2009; 421:145-156.

4 Berridge MJ. Rapid accumulation of inositol trisphosphate reveals that agonists hydrolyse polyphosphoinositides instead of phosphatidylinositol. Biochem J 1983; 212:849-858.

5 Meijer HJ, Munnik T. Phospholipid-based signaling in plants. Annu Rev Plant Biol 2003; 54:265-306.

6 Wasteneys GO, Galway ME. Remodeling the cytoskeleton for growth and form: an overview with some new views. Annu Rev Plant Biol 2003; 54:691-722.

7 Wenk MR, De Camilli P. Protein-lipid interactions and phosphoinositide metabolism in membrane traffic: Insights from vesicle recycling in nerve terminals. Proc Natl Acad Sci USA 2004; 101:8262-8269.

8 Cote GG, Yueh YG, Crain RC. Phosphoinositide turnover and its role in plant signal transduction. Subcell Biochem 1996; 26:317-343.

9 Suh BC, Hille B. Regulation of ion channels by phosphatidylinositol 4,5-bisphosphate. Curr Opin Neurobiol 2005; 15:370-378.

10 Haucke V. Phosphoinositide regulation of clathrin-mediated endocytosis. Biochem Soc Trans 2005; 33:1285-1289.

11 Clague MJ, UrbéS, de Lartigue J. Phosphoinositides and the endocytic pathway. Exp Cell Res 2009; 315:1627-1631.

12 Yao HY, Xue HW. Signals and mechanisms affecting vesicular trafficking during root growth. Curr Opin Plant Biol 2011 Jul 15. doi:10.1016/j.pbi.2011.06.009

13 Mueller-Roeber B, Pical C. Inositol phospholipid metabolism in Arabidopsis. Characterized and putative isoforms of inositol phospholipid kinase and phosphoinositide-specific phospholipase C. Plant Physiol 2002; 130:22-46.

14 Nishi M, Mizushima A, Nakagawara K, Takeshima H. Characterization of human junctophilin subtype genes. Biochem Biophys Res Commun 2000; 273:920-927.

15 Elge S, Brearley C, Xia HJ, Kehr J, Xue HW, Mueller-Roeber B. An Arabidopsis inositol phospholipid kinase strongly expressed in procambial cells: synthesis of $\operatorname{PtdIns}(4,5) \mathrm{P}_{2}$ and PtdIns $(3,4,5) \mathrm{P}_{3}$ in insect cells by 5-phosphorylation of precursors. Plant J 2001; 26:561-571.

16 Stenzel I, Ischebeck T, König S, et al. The type B phosphatidylinositol-4-phosphate 5-kinase 3 is essential for root hair formation in Arabidopsis thaliana. Plant Cell 2008; 20:124141.

17 Ischebeck T, Stenzel I, Heilmann I. Type B phosphatidylinositol-4-phosphate 5-kinases mediate Arabidopsis and Nicotiana tabacum pollen tube growth by regulating apical pectin secretion. Plant Cell 2008; 20:3312-3330. 
18 Zhao Y, Yan A, Feijo J, et al. Phosphoinositides regulate clathrin-dependent endocytosis at the tip of pollen tubes in Arabidopsis and Tobacco. Plant Cell 2010; 22:4031-4044.

19 Mikami K, Katagiri T, Iuchi S, Yamaguchi-Shinozaki K, Shinozaki K. A gene encoding phosphatidylinositol-4-phosphase 5-kinase is induced by water stress and abscisic acid in Arabidopsis thaliana. Plant J 1998; 15:563-568.

20 Kusano H, Testerink C, Vermeer JEM, et al. The Arabidopsis phosphatidylinositol phosphate 5-kinase PIP5K3 is a key regulator of root hair tip growth. Plant Cell 2008; 20:367-380.

21 Lee Y, Kim YW, Jeon BW, et al. Phosphatidylinositol 4, 5-bisphosphate is important for stomatal opening. Plant $J$ 2007; 52:803-816.

22 Sousa E, Kost B, Malhó R. Arabidopsis phosphatidylinositol4-monophosphate 5-kinase 4 regulates pollen tube growth and polarity by modulating membrane recycling. Plant Cell 2008; 20:3050-3064.

23 Lou Y, Gou JY, Xue HW. PIP5K9, an Arabidopsis phosphatidylinositol monophosphate kinase, interacts with a cytosolic invertase to negatively regulate sugar-mediated root growth. Plant Cell 2007; 19:163-181.

24 Casimiro I, Marchant A, Bhalerao RP, et al. Auxin transport promotes Arabidopsis lateral root initiation. Plant Cell 2001; 13:843-852.

25 Nibau C, Gibbs DJ, Coates JC. Branching out in new directions: the control of root architecture by lateral root formation. New Phytol 2008; 179:595-614.

26 Boerjan W, Cervera MT, Delarue M, et al. Superroot, a recessive mutation in Arabidopsis, confers auxin overproduction. Plant Cell 1995; 7:1405-1419.

27 King JJ, Stimart DP, Fisher RH, Bleecker AB. A mutation altering auxin homeostasis and plant morphology in Arabidopsis. Plant Cell 1995; 7:2023-2037.

28 Laskowski MJ, Williams ME, Nusbaum HC, Sussex IM. Formation of lateral root meristems is a two-stage process. Development 1995; 121:3303-3310.

29 Ruegger M, Dewey E, Gray WM, Hobbie L, Turner J, Estelle M. The TIR1 protein of Arabidopsis functions in auxin response and is related to human SKP2 and yeast Grr1p. Genes Dev 1998; 12:198-207.

30 Marchant A, Bhalerao R, Casimiro I, et al. AUX1 promotes lateral root formation by facilitating indole-3-acetic acid distribution between sink and source tissues in the Arabidopsis seedling. Plant Cell 2002; 14:589-597.

31 Park JE, Park JY, Kim YS, et al. GH3-mediated auxin homeostasis links growth regulation with stress adaptation response in Arabidopsis. J Biol Chem 2007; 282:10036-10046.

32 Swarup K, Benkova E, Swarup R, et al. The auxin influx carrier LAX3 promotes lateral root emergence. Nat Cell Biol 2008; 10:946-954.

33 Bandyopadhyay A, Blakeslee JJ, Lee OR, et al. Interactions of PIN and PGP auxin transport mechanisms. Biochem Soc Trans 2007; 35:137-141.

34 Petrasek J, Mravec J, Bouchard R, et al. PIN proteins perform a rate-limiting function in cellular auxin efflux. Science 2006; 312:914-918.

35 Zhang J, Vanneste S, Brewer PB, et al. Inositol trisphosphateinduced $\mathrm{Ca} 2+$ signaling modulates auxin transport and PIN polarity. Dev Cell 2011; 14:855-66.
36 Bennett MJ, Marchant A, Green HG, et al. Arabidopsis AUX1 gene: a permease-like regulator of root gravitropism. Science 1996; 273:948-950.

37 Müller A, Guan C, Gälweiler L, et al. AtPIN2 defines a locus of Arabidopsis for root gravitropism control. EMBO J 1998; 17:6903-6911.

38 Li L, Xu J, Xu ZH, Xue HW. Brassinosteroids stimulate plant tropisms through modulation of polar auxin transport in Brassica and Arabidopsis. Plant Cell 2005; 17:2738-2753.

39 Friml J, Wisniewska J, Benkova E, Mendgen K, Palme K. Lateral relocation of auxin efflux regulator PIN3 mediates tropism in Arabidopsis. Nature 2002; 415:806-809.

40 Perera IY, Heilmann I, Boss WF. Transient and sustained increases in inositol 1,4,5-trisphosphate precede the differential growth response in gravistimulated maize pulvini. Proc Natl Acad Sci USA 1999; 96:5838-5843.

41 Perera IY, Hung CY, Brady S, Muday GK, Boss WF. A universal role for inositol 1,4,5-trisphosphate-mediated signaling in plant gravitropism. Plant Physiol 2006; 140:746-760.

42 Wang Y, Lin WH, Chen X, Xue HW. The role of Arabidopsis 5PTase 13 in root gravitropism through modulation of vesicle trafficking. Cell Res 2009; 19:1191-1204.

43 Geldner N, Anders N, Wolters H, et al. The Arabidopsis GNOM ARF-GEF mediates endosomal recycling, auxin transport, and auxin-dependent plant growth. Cell 2003; 112:219230 .

44 Jaillais Y, Fobis-Loisy I, Miege C, Rollin C, Gaude T. AtSNX1 defines an endosome for auxin-carrier trafficking in Arabidopsis. Nature 2006; 443:106-109.

45 Dhonukshe P, Aniento F, Hwang I, et al. Clathrin-mediated constitutive endocytosis of PIN auxin efflux carriers in Arabidopsis. Curr Biol 2007; 17:520-527.

46 Geldner N, Friml J, Stierhof YD, Jurgens G, Palme K. Auxin transport inhibitors block PIN1 cycling and vesicle trafficking. Nature 2001; 413:425-428.

47 Men S, BouttéY, Ikeda Y, et al. Sterol-dependent endocytosis mediates post-cytokinetic acquisition of PIN2 auxin efflux carrier polarity. Nat Cell Biol 2008; 10:237-244.

48 Pan J, Fujioka S, Peng J, Chen J, Li G, Chen R. The E3 ubiquitin ligase $\mathrm{SCF}^{\mathrm{TIR} 1 / \mathrm{AFB}}$ and membrane sterols play key roles in auxin regulation of endocytosis, recycling, and plasma membrane accumulation of the auxin efflux transporter PIN2 in Arabidopsis thaliana. Plant Cell 2009; 21:568-580.

49 Lin WH, Wang Y, Mueller-Roeber B, Brearley CA, Xu ZH, Xue HW. At5PTase13 modulates cotyledon vein development through regulating auxin homeostasis. Plant Physiol 2005; 139:1677-1691.

50 Li G, Xue HW. Arabidopsis PLDל2 regulates vesicle trafficking and is required for auxin response. Plant Cell 2007; 19:281-295.

51 Zhang ZB, Yang G, Arana F, Chen Z, Li Y, Xia HJ. Arabidopsis inositol polyphosphate 6-/3-kinase (AtIpk2b) is involved in axillary shoot branching via auxin signaling. Plant Physiol 2007; 144:942-951.

52 Alonso JM, Stepanova AN, Leisse TJ, et al. Genome-wide insertional mutagenesis of Arabidopsis thaliana. Science 2003; 301:653-657.

53 Zhang H, Jennings A, Barlow PW, Forde GB. Dual pathways for regulation of root branching by nitrate. Proc Natl Acad Sci 
USA 1999; 96:6529-6534.

54 DeWald DB, Ozaki S, Malaviya S, et al. Cellular calcium mobilization in response to phosphoinositide delivery. Cell Calcium 2005; 38:59-72.

55 Casimiro I, Beeckman T, Graham N, et al. Dissecting Arabidopsis lateral root development. Trends Plant Sci 2003; 8:165-171.

56 Ulmasov T, Murfett J, Hagen G, Guilfoyle TJ. Aux/IAA proteins repress expression of reporter genes containing natural and highly active synthetic auxin response elements. Plant Cell 1997; 9:1963-1971.

57 Rashotte AM, Brady SR, Reed RC, Ant SJ, Muday GK. Basipetal auxin transport is required for gravitropism in roots of Arabidopsis. Plant Physiol 2000; 122:481-490.

58 Perrin RM, Young LS, Murthy UMN, et al. Gravity signal transduction in primary roots. Ann Bot 2005; 96:737-743.

59 Palme K, Dovzhenko A, Ditengou FA. Auxin transport and gravitational research: perspectives. Protoplasma 2006; 229:175-181.

60 Owen DJ, Collins BM, Evans PR. Adaptors for clathrin coats: structure and function. Annu Rev Cell Dev Biol 2004; 20:153191.

61 Bolte S, Talbot C, Boutte Y, Catrice O, Read ND, SatiatJeunemaitre B. FM-dyes as experimental probes for dissecting vesicle trafficking in living plant cells. J Microsc 2004; 214:159-173.

62 Nebenfuhr A, Ritzenthaler C, Robinson DG. Brefeldin A: Deciphering an enigmatic inhibitor of secretion. Plant Physiol 2002; 130:1102-1108.

63 Blakeslee JJ, Bandyopadhyay A, Lee OR, et al. Interactions among PIN-FORMED and P-glycoprotein auxin transporters in Arabidopsis. Plant Cell 2007; 1:131-147.

64 Rashotte A, Delong A, Muday G. Genetic and chemical reductions in protein phosphatase activity alter auxin transport, gravity response, and lateral root growth. Plant Cell 2001; 13:1683-1697.

65 Boonsirichai K, Sedbrook JC, Chen R, Gilroy S, Masson PH. ALTERED RESPONSE TO GRAVITY is a peripheral membrane protein that modulates gravity-induced cytoplasmic alkalinization and lateral auxin transport in plant statocytes. Plant Cell 2003; 15:2612-2625.

66 Ottenschläger I, Wolff P, Wolverton C, et al. Gravity-regulated differential auxin transport from columella to lateral root cap cells. Proc Natl Acad Sci USA 2003; 100:2987-2991.

67 Yang X, Song L, Xue HW. Membrane steroid binding protein 1 (MSBP1) stimulates tropism by regulating vesicle trafficking and auxin redistribution. Mol Plant 2008; 1:1077-1087.

68 Abas L, Benjamins R, Malenica N, et al. Intracellular trafficking and proteolysis of the Arabidopsis auxin-efflux facilitator PIN2 are involved in root gravitropism. Nat Cell Biol 2006; 8:249-256.

69 Camacho L, Smertenko AP, Pérez-Gómez J, Hussey PJ, Moore I. Arabidopsis Rab-E GTPases exhibit a novel interaction with a plasma-membrane phosphatidylinositol-4phosphate 5-kinase. J Cell Sci 2009; 122:4383-4392.

70 Speth EB, Imboden L, Hauck P, He SY. Subcellular localization and functional analysis of the Arabidopsis GTPase RabE. Plant Physiol 2009; 149:1824-1837.

71 Benková E, Michniewicz M, Sauer M, et al. Local, efflux-dependent auxin gradients as a common module for plant organ formation. Cell 2003; 115:591-602.

72 Di Paolo G, De Camilli P. Phosphoinositides in cell regulation and membrane dynamics. Nature 2006; 443:651-657.

73 Clough SJ, Bent AF. Floral dip: A simplified method for Agrobacterium-mediated transformation of Arabidopsis thaliana. Plant $J$ 1998; 16:735-743.

74 Liu W, Xu ZH, Luo D, Xue HW. Roles of OsCKI1, a rice casein kinase I, in root development and plant hormone sensitivity. Plant J 2003; 36:189-202.

75 Kowalczyk M, Sandberg G. Quantitative analysis of indole3-acetic acid metabolites in Arabidopsis. Plant Physiol 2001; 127:1845-1853.

76 Qi J, Qian Q, Bu Q, et al. Mutation of the rice Narrow leaf1 gene, which encodes a novel protein, affects vein patterning and polar auxin transport. Plant Physiol 2008; 147:19471959.

(Supplementary information is linked to the online version of the paper on the Cell Research website.) 